\title{
Evidence for widespread selection in shaping the genomic landscape during speciation of Populus
}

Jing Wang ${ }^{1 *}$, Nathaniel R. Street ${ }^{2}$, Eung-Jun Park ${ }^{3}$, Jianquan Liu ${ }^{1}$, Pär K. Ingvarsson ${ }^{4}$

${ }^{1}$ Key Laboratory for Bio-resources and Eco-environment, College of Life Science, Sichuan University, Chengdu, China

${ }^{2}$ Umeå Plant Science Centre, Department of Plant Physiology, Umeå University, 90187 Umeå, Sweden

3 Department of Bioresources, National Institute of Forest Science, Suwon 16631, Republic of Korea

4 Department of Plant Biology, Uppsala BioCenter, Swedish University of Agricultural Sciences, PO Box 7080, 75007, Uppsala, Sweden

*Correspondence: wangjing2019@scu.edu.cn

Running title: Genomic impact of selection in Populus 


\section{Abstract}

3 Increasing our understanding of how various evolutionary processes drive the genomic

4 landscape of variation is fundamental to a better understanding of the genomic

5 consequences of speciation. However, the genome-wide patterns of within- and

6 between- species variation have not been fully investigated in most forest tree species

7 despite their global ecological and economic importance. Here, we use whole-genome

8 resequencing data from four Populus species spanning the speciation continuum to

9 reconstruct their demographic histories, investigate patterns of diversity and divergence,

10 infer their genealogical relationships and estimate the extent of ancient introgression

11 across the genome. Our results show substantial variation in these patterns along the

12 genomes although this variation is not randomly distributed but is strongly predicted by

13 the local recombination rates and the density of functional elements. This implies that

14 the interaction between recurrent selection and intrinsic genomic features has

15 dramatically sculpted the genomic landscape over long periods of time. In addition, our

16 findings provide evidence that, apart from background selection, recent positive

17 selection and long-term balancing selection are also crucial components in shaping

18 patterns of genome-wide variation during the speciation process.

20 Keywords: Linked selection, Recombination, Incomplete lineage sorting, Phylogenetic

21 relationship, Ancient introgression, Populus 


\section{Introduction}

24 Determining the evolutionary forces affecting patterns of genome-wide variation has

25 been a central goal in evolutionary biology over the past several decades (Seehausen et

26 al., 2014). Furthermore, studying variation in levels of differentiation within and

27 between closely related species has the potential to yield important insights into the

28 process of speciation (Ravinet et al., 2017; Wolf \& Ellegren, 2017). Studies in a broad

29 range of taxonomic groups have revealed a picture of a highly heterogeneous genomic

30 landscape with peaks and valleys of diversity and differentiation (Han et al., 2017;

31 Nadeau et al., 2012; Stankowski et al., 2019; Turner, Hahn, \& Nuzhdin, 2005). Local

32 peaks of elevated divergence are usually referred to as 'speciation islands' and are

33 thought to represent regions that drive the reproductive isolation between incipient

34 species (Abbott et al., 2013; Wu, 2001). Between these islands, gene flow acts to

35 homogenize the reminder of genome and hence acts to limit differentiation (Feder,

36 Egan, \& Nosil, 2012; Nosil, Funk, \& Ortiz - Barrientos, 2009). However, a plethora of

37 recent studies highlight that the heterogeneous patterns of differentiation can evolve

38 through processes that are unrelated to speciation per se (Burri et al., 2015; Cruickshank

$39 \&$ Hahn, 2014). For example, even in the absence of gene flow, natural selection, in the

40 form of either a selective sweep or background selection, can cause reduced genetic

41 diversity not only at the target sites under selection but also at linked neutral sites (Han

42 et al., 2017; Phung, Huber, \& Lohmueller, 2016). Such selection could accelerate

43 lineage sorting and will hence inevitably result in increased genetic differentiation

44 between species in these regions (Burri, 2017). Furthermore, the long-term action of

45 linked selection in ancestral as opposed to extant lineages can also affect the amount

46 and distribution of ancestral polymorphisms (Ma et al., 2018; Munch, Nam, Schierup, 
47 \& Mailund, 2016; Scally et al., 2012), which can further result in heterogeneous

48 patterns of genealogical relationships among closely related species (Mailund, Munch,

49 \& Schierup, 2014; Pease \& Hahn, 2013). Despite widespread interest in speciation

50 genomics, there remains little consensus as to how various evolutionary processes have

51 shaped the genomic landscape during the speciation process that eventually gives rise to

52 new species (Ravinet et al., 2017).

53 Empirical studies suggest that the formation of the genomic landscape of

54 diversity during speciation is highly influenced by the demographic histories of the

55 species, the types of selection acting on different genomic regions and also several other

56 intrinsic genomic features (Burri, 2017; Ellegren \& Galtier, 2016). Disentangling the

57 effects of speciation (i.e. species split time, strict isolation or divergence with gene flow)

58 is important for interpreting the patterns of genome-wide variation, because without a

59 clear picture of the demographic history of the descendant species, it is challenging to

60 distinguish whether heterogenous genomic differentiation arose due to genetic drift,

61 local adaptation or introgression (Nadachowska-Brzyska et al., 2013; Ravinet et al.,

62 2018). Furthermore, as the speciation process advances, the evolution of genome-wide

63 patterns of variation can be influenced by different forms of selection (Cutter \& Payseur,

64 2013). Under a background selection model, purifying selection continuously eliminates

65 deleterious mutations, resulting in reduced levels of genetic diversity at linked loci and

66 increased levels of $F_{\text {ST }}$ (a relative measure of genetic divergence) (Charlesworth, 2012;

67 Charlesworth, Morgan, \& Charlesworth, 1993; Hudson \& Kaplan, 1995). Under a

68 selective sweep model, genetic variants linked to beneficial mutations acted upon by

69 positive selection hitchhike along and reach high frequency (Kaplan, Hudson, \&

70 Langley, 1989; Smith \& Haigh, 1974). Accordingly, even in the absence of gene flow,

71 selection due to, for instance local ecological adaptation, can result in reduced diversity 
72 and increased $F_{\text {ST }}$ (Cruickshank \& Hahn, 2014). In comparison to purifying and positive

73 selection, long-term balancing selection favors the maintenance of advantageous

74 polymorphisms for many generations, which instead result in genomic regions with

75 elevated genetic diversity and reduced $F_{\mathrm{ST}}$ (Charlesworth, 2006; Guerrero \& Hahn,

76 2017). As deleterious mutations are assumed to be much more common compared to

77 beneficial mutations, background selection has been argued to play a major role in the

78 evolution of diversity (Burri, 2017; Lohmueller et al., 2011; Phung et al., 2016).

79 However, many recent simulation studies have shown that background selection alone

80 is far from sufficient for generating the heterogenous genomic landscapes observed in

81 empirical studies of recently diverged species, and other evolutionary processes (such as

82 positive selection) are thus required to explain the observed patterns (Matthey - Doret

83 \& Whitlock 2019; Stankowski et al., 2019).

84 Regardless of the role of demographic processes and selection, genomic

85 features, such as recombination rate variation and the heterogeneous density of

86 functional sites, are also expected to play key roles in mediating the efficacy and extent

87 of selection and gene flow, as well as how these processes interact as the speciation

88 process proceeds (Flaxman, Wacholder, Feder, \& Nosil, 2014; Hurst, Pál, \& Lercher,

89 2004; Nachman \& Payseur, 2012). Local rates of recombination interacts with natural

90 selection and are known to have a profound effect on patterns of genomic diversity,

91 incomplete lineage sorting (ILS) and rates of introgression (Begun \& Aquadro, 1992;

92 Comeron, Williford, \& Kliman, 2008; Cutter \& Payseur, 2013). Independent of the

93 recombination rate, the density of functional sites can also influence genome-wide

94 patterns of diversity since functional regions are more likely to experience either

95 stronger effects of positive or purifying selection compared to nonfunctional regions

96 where mutations are assumed to have little effect on fitness (Al-Shahrour et al., 2010; 
97 Nordborg et al., 2005). The long-term diversity-reducing effects of selection in

98 functional regions will reduce locally effective population size $\left(N_{\mathrm{e}}\right)$, accelerate lineage

99 sorting and increase genetic divergence between species (Flowers et al., 2011; Hobolth,

100 Dutheil, Hawks, Schierup, \& Mailund, 2011). As it becomes increasingly feasible to

101 generate whole genome resequencing data from closely related species, the importance

102 of conserved genomic features in shaping the topography of the genomic landscape of

103 speciation has increasingly been highlighted by several studies in a diverse set of taxa

104 showing highly correlated patterns of differentiation among independently species pairs

105 (Burri, 2017; Delmore et al., 2018; Van Doren et al., 2017; Vijay et al., 2017).

106 Forest trees provide an excellent system to address the genomic architecture of 107 adaptation and speciation in natural populations because they are mostly 108 undomesticated without much anthropogenic influence, ecologically important across a 109 wide variety of habitats and harbour abundant genetic and phenotypic variation (Neale 110 \& Ingvarsson, 2008; Neale \& Kremer, 2011). In this study, we focus on four Populus 111 species (Populus tremula, P. davidiana, P. tremuloides and P. trichocarpa) that span 112 the speciation continuum. All four species are all deciduous, obligated outcrossing tree 113 species that have wide geographical distributions throughout the Northern Hemisphere 114 (Figure 1A). Among them, P. tremula (European aspen), P. davidiana (Chinese aspen) 115 and $P$. tremuloides (American aspen) are sibling aspen species belonging to the same 116 section of the genus Populus (section Populus) (Eckenwalder, 1996; Hamzeh \& 117 Dayanandan, 2004). Earlier phylogenetic studies have revealed that $P$. tremuloides 118 diverged from the other two species following the break-up of the Bering Land bridge, 119 whereas the uplift of the Qinghai-Tibetan Plateau and the associated climate oscillations 120 may have driven the divergence between $P$. tremula and P. davidiana (Du et al., 2015). 121 In addition, these aspen species can readily hybridize and their artificial hybrids show 
122 heterosis for many growth and wood characteristics (Hart, De Araujo, Thomas, \&

123 Mansfield, 2013), suggesting that the speciation process has not gone to completion

124 among the three aspen species. In comparison, $P$. trichocarpa belongs to a different

125 section of the genus Populus (section Tacamahaca), and it is reproductively isolated

126 from all aspen species (Jansson \& Douglas, 2007). Facilitated by the availability of a

127 high-quality reference genome of P. trichocapra (Tuskan et al., 2006), the four Populus

128 species represent a promising model system to investigate how various evolutionary

129 forces have shaped the evolution of the genomic landscape of differentiation across the

130 speciation continuum in forest trees.

131 We use whole-genome re-sequencing in the four Populus species to (i)

132 determine their speciation history and characterize whether there is historical gene flow

133 between the now-allopatric species; (ii) examine the fine-scale genomic landscapes of

134 diversity and divergence across species at different stages of divergence; (iii) quantify

135 the extent of genome-wide genealogical discordance and ancient hybridization among

136 the three closely related aspen species; (iv) identify the signatures of positive selection

137 and long-term balancing selection along the genome, and uncover how they impact

138 levels of variation during speciation. Overall, our main aim is to disentangle and

139 understand how the multitude of evolutionary processes have shaped the genomic

140 architecture during speciation.

142 2. Materials and Methods

144 2.1 Sample collection, whole-genome resequencing and genotype calling

145 We used whole genome resequencing data from eight individuals each of Populus 146 tremula, P. tremuloides and P. trichocarpa, as described in Wang et al. (2016a), and 
147 additional eight individuals of $P$. davidiana that are first reported in this study (Table

148 S1). The sampling was from a single geographic region for each species (Figure 1A).

149 Briefly, sequencing of all samples was carried out on the Illumina HiSeq 2000 platform.

150 Prior to read mapping, we used Trimmomatic (Lohse et al., 2012) to remove adapter

151 sequences and to trim low quality bases from the start or the end of reads (base quality $\leq$

152 20). If the processed reads were shorter than 36 bases after trimming, the entire reads

153 were discarded. After quality control, we mapped the remaining reads from each

154 individual to the $P$. trichocarpa reference genome (v3.0) (Tuskan et al., 2006) using

155 BWA-MEM algorithm with default parameters, as implemented in bwa-0.7.10 (Li, 156 2013).

157 To minimize the influence of mapping bias, several further filtering steps were 158 employed before genotype calling. First, we used RealignerTargetCreator and 159 IndelRealigner in GATK v3.8.0 (DePristo et al., 2011) to correct for the mis-alignment 160 of bases in regions around insertions and/or deletions (indels). Second, to account for 161 the artifacts due to PCR duplication introduced during library construction, we used the 162 MarkDuplicates method from Picard packages (http://broadinstitute.github.is/picard/) to 163 only retain the read or read-pair with the highest summed base quality among those with 164 identical external coordinates and same insert lengths. Additionally, we further 165 discarded site types that likely cause mapping bias based on three criteria: (1) those with 166 extreme read coverage (less then $4 \times$ or higher than twice of the mean coverage); (2) 167 covered by more than two reads of mapping score equaling zero per individual; (3) 168 overlapping known repetitive elements as identified by RepeatMasker (Tarailo -

169 Graovac \& Chen, 2009). Finally, sites that passed all these filtering criteria were used in 170 downstream analyses. This left a total of $168,950,389$ sites for further analysis $(42.8 \%$ 171 of collinear genomic sequences of the $P$. trichocarpa genome assembly). 
172 After filtering, we implemented two complementary approaches for genotype

173 calling. First, to account for the bias inherent in genotype calling approach from next 174 generation sequencing (NGS) data (Nielsen, Korneliussen, Albrechtsen, Li, \& Wang,

175 2012), the population genetic estimates that relied on site frequency spectrum (SFS)

176 were calculated using ANGSD v0.917 (Korneliussen, Albrechtsen, \& Nielsen, 2014).

177 Second, for the analyses that require accurate single nucleotide polymorphism (SNP)

178 calls, genotype calling in each individual was performed using HaplotypeCaller of the

179 GATK v3.8.0, and GenotypeGVCFs was then used to merge multi-sample records from

180 the four species together for re-genotyping and re-annotation of the newly merged VCF

181 (DePristo et al., 2011). To minimize genotype calling bias and to retain high-quality

182 SNPs, we further performed several filtering steps: (1) SNPs that overlapped with sites

183 not passing all previous filtering criteria were removed; (2) only bi-allelic SNPs with a

184 distance of at least 5 bp away from any indels were retained; (3) genotypes with read

185 depth (DP) $<5$ and/or with genotype quality score (GQ) $<10$ were treated as missing,

186 and we then removed all SNPs with a genotype missing rate $>10 \%$. After all these steps

187 of filtering, a total of $8,568,990$ SNPs were retained across the four Populus species.

188 For the analyses that required imputed and phased dataset, BEAGLE v4.1 (Browning \&

189 Browning, 2009) was used to infer haplotypes of individuals within each species.

\subsection{Phylogenetic relationships and population structure analysis}

\section{Chloroplast phylogeny}

193 To infer the phylogenetic relationship of the four Populus species based on chloroplast

194 data, we first mapped the filtered reads from our resequencing data against the $P$.

195 trichocarpa chloroplast genome using bwa-aln 0.7.10 (Li \& Durbin, 2009). Then,

196 UnifiedGenotyper in GATK v3.8.0 was used to call SNPs at all sites (--output_mode 
197 EMIT_ALL_SITES). Since chloroplasts are haploid and SNPs are thus expected to be

198 homozygous, the haploid option (-ploidy 1) in UnifiedGenotyper was used. After

199 treating sites with GQ $<30$ as missing data, only bi-allelic SNPs with quality by depth

200 (QD) $\geq 10$ and with a missing rate $\leq 20 \%$ were retained. Finally, a consensus tree was

201 constructed based on 1,292 chloroplast SNPs using maximum likelihood method

202 implemented in SNPhylo (Lee, Guo, Wang, Kim, \& Paterson, 2014).

204 Principle component analysis (PCA)

205 To account for the uncertainty in genotype calls, PCA was performed using ANGSD 206 v0.917 and ngsTools v1.0.1 (Fumagalli, Vieira, Linderoth, \& Nielsen, 2014). We first

207 used the SAMTools model (Li et al., 2009) in ANGSD to estimate genotype likelihoods

208 from BAM files using only reads with a minimal base quality score of 20 and a minimal

209 mapping quality score of 30 across all individuals. ngs Tools was then used to compute

210 the expected covariance matrix across pairs of individuals for the four species based on

211 the genotype posterior probabilities across all filtered sites. Eigenvectors and

212 eigenvalues were generated with the $\mathrm{R}$ function eigen from the covariance matrix, and

213 the significance level was determined using the Tracy-Widom test as implemented in

214 EIGENSOFT version 6.1.4 (Patterson, Price, \& Reich, 2006).

216 Identity-by-descent (IBD) blocks analysis

217 To determine the extent to which individuals across the four species shared DNA 218 segments, the identity-by-descent block analysis was performed for the four species 219 using BEAGLE v4.1 (Browning \& Browning, 2013) with the following parameters:

220 window $=100,000 ;$ overlap $=10,000 ;$ ibdtrim $=100 ;$ ibdlod $=5$. 


\subsection{Demographic history reconstruction}

\section{$223 \quad M S M C$}

224 We used Multiple Sequentially Markovian Coalescent approach (MSMC v2) (Schiffels

225 \& Durbin, 2014) to infer patterns of historical patterns of effective population sizes

226 changes through time for all four Populus species. Only sites passing all above filtering

227 criteria were included in analyses. Because different number of individuals and

228 haplotypes provides different resolution for recent and distant population histories, we

229 applied MSMC to phased whole-genome sequences from one (two haplotypes, which

230 can infer more distant size changes), two (four haplotypes, which infer size changes at

231 intermediate time scales) and four (eight haplotypes, which infer the most recent size

232 changes) individuals for each species, respectively. We did not include more haplotypes

233 due to the computational cost of using larger haplotype sets. In total, we have 8, 28 and

23470 different individual configurations for two-, four-, and eight- haplotype analyses in

235 each species. We ran MSMC on all individual configurations and estimated medians

236 and standard deviations of effective population sizes changes across time. To convert

237 the coalescent scaled time to absolute time in years, we used a mutation rate of $2 \times 10^{-9}$

238 per site per year (Koch, Haubold, \& Mitchell-Olds, 2000) and a generation time of 15 239 years.

\section{$241 \quad$ Fastsimcoal2}

242 Given the long divergence time and the low number of polymorphic sites shared 243 between aspens and P. trichocarpa (Wang, Street, Scofield, \& Ingvarsson, 2016a), we 244 used a coalescent simulation-based method implemented in fastsimcoal2.6 (Excoffier, 245 Dupanloup, Huerta-Sánchez, Sousa, \& Foll, 2013) to only infer the demographic and 246 speciation histories only for the three aspen species. For all possible pairs of the three 
247 species, the two-dimensional joint SFS (2D-SFS) was constructed from the posterior 248 probabilities of sample allele frequencies using ANGSD v0.917 (Figure S1). A total of 249 twenty-nine models were evaluated and all models began with the split of an ancestral 250 population into the Eurasia and the North America lineage ( $P$. tremuloides) followed by 251 the split of the Eurasian lineage into P. tremula and $P$. davidiana. The models differed 252 in terms of (1) whether post-divergence gene flow was present or not, (2) time, level 253 and pattern of gene flow between the three aspen species, and (3) the occurrence and 254 pattern of population expansion in $P$. tremuloides given a genome-wide excess of rare 255 frequency alleles that we observed in this species in our previous study (Wang et al., 256 2016a) and also in this study (Figure S2). Alternative demographic models were fitted 257 to the joint SFS data. The global maximum likelihood estimates for all demographic 258 parameters under each model were obtained from 50 independent runs, with 100,000 259 coalescent simulations per likelihood estimates (-n 100000, -N 100000) and 40 cycles of 260 the likelihood maximization algorithm. The models were compared based on the 261 maximum value of likelihood over the 50 independent runs using the Akaike's weight 262 calculated following Excoffier et al. (2013). The model with the maximum Akaike's 263 weight value was chosen as the optimal one. Confidence intervals were generated by 264 performing parametric bootstrapping with 100 bootstrap replicates, and with 50 265 independent runs in each bootstrap. As for MSMC, we assumed a mutation rate of $2662 \times 10^{-9}$ per site per year and a generation time of 15 years (Koch et al., 2000) when 267 converting estimates to units of years and individuals.

\subsection{Intra- and inter- species summary statistics}

$270 \quad$ Intra-species genomic diversity 
271 For each species, based on the SAMTools genotype likelihood model (Li et al., 2009),

272 we used ANGSD v0.917 (Korneliussen et al., 2014) to estimate allele frequency

273 likelihoods, obtain a maximum likelihood estimate of the folded site frequency

274 spectrum and used this to calculate nucleotide diversity $(\pi)$ in non-overlapping sliding

275 windows of $10 \mathrm{Kbp}$ and $100 \mathrm{Kbp}$ across the entire genome. Only sites with a minimum

276 mapping quality of 30 and minimum base quality of 20 were used in the estimation.

277 Windows were discarded if there were less than $10 \%$ sites left after all of the filtering

278 steps described above. Since our previous study showed that linkage disequilibrium

279 (LD) decays within $10 \mathrm{Kbp}$ in different species of Populus (Wang et al., 2016a), in the

280 following we focused more on estimates derived from $10 \mathrm{Kbp}$ windows.

\section{Inter-species genomic divergence}

283 For each species pair, we estimated two divergence metrics across the $10 \mathrm{Kbp}$ and 100

284 Kbp non-overlapping windows: genetic differentiation $\left(F_{\mathrm{ST}}\right)$ and sequence divergence

$285\left(d_{x y}\right)$. Without relying on SNP or genotype calling (Fumagalli et al., 2013), we first used

286 ANGSD to calculate posterior probabilities of sample allele frequency for each species.

287 Then, the program ngsFST from the ngsTools package was used to estimate $F_{\mathrm{ST}}$

288 between species using a method-of-moments estimator, and the program ngs Stat was

289 used to calculate $d_{x y}$ between species at each site. Finally, we averaged these divergence

290 values across all sites within each window.

Population-scaled recombination rate

293 For each species we used LDhelmet v1.9 (Chan, Jenkins, \& Song, 2012), a

294 coalescent-based, reversible-jump Markov chain Monte Carlo (rjMCM) simulation

295 method, to estimate the population-scaled recombination rate, $\rho$. First, VCFtools 
296 (Danecek et al., 2011) and custom shell script were used to tailor the phased genotype

297 data of each chromosome to the necessary input sequence file (fasta format). Then, we

298 used 'find_confs' in LDhelmet to concatenate all the input sequences files and generate

299 a haplotype configuration file per species. Thereafter, 'table_gen' was used to compute

300 the likelihood lookup table for each species, where we assume the approximate

301 genome-wide neutral diversity $(\theta)$ of 0.01 for the three aspen species and of 0.005 for $P$.

302 trichocarpa (Wang et al., 2016a), and the grid of $\rho$ values was specified as $-\mathrm{r} 0.00 .1$

30310.01 .0100 .0 for all species. In addition, the optional 'pade' component of LDhelmet

304 was included in the analysis, which computes the Padé coefficients (-x 11) from the

305 haplotype configuration file. Finally, we ran LDhelmet with window size of 50 SNPs

306 and block penalty of 50 for a total of $1,000,000$ iterations, discarding the first 100,000

307 as burn-in. We then calculated weighted average of the estimated $\rho$ in $10 \mathrm{Kbp}$ and 100

$308 \mathrm{Kbp}$ windows, respectively. Windows with less than 50 SNPs (for $10 \mathrm{Kbp}$ windows)

309 and $200 \mathrm{SNPs}$ (for $100 \mathrm{Kbp}$ windows) left from previous filtering steps were discarded.

\subsection{Window-based phylogenomic analysis}

312 Topology weighting

313 As expected for a clade with rapid radiation, genealogies may vary widely across

314 different genomic regions (Lamichhaney et al., 2015). Given that $P$. trichocarpa is

315 distantly related from the other three aspen species (Hamzeh \& Dayanandan, 2004), we

316 used Twisst, a topology weighting method by iterative sampling of subtrees (Martin \&

317 Van Belleghem, 2017), to assess and quantify the phylogenetic discordance among the

318 three aspen species along the genome. The genealogical relationships of these species

319 can be defined by three possible topologies: $[(P$. tremula, $P$. davidiana $), P$.

320 tremuloides $],[(P$. tremula, $P$. tremuloides $), P$. davidiana $],[(P$. davidiana, $P$. 
321 tremuloides), P. tremula]. Using $P$. trichocarpa as the outgroup species, local

322 phylogenetic subtrees was inferred in RAxML v8.2.4 (Stamatakis, 2014) with the

323 GTRCATI model over non-overlapping $10 \mathrm{Kbp}$ and $100 \mathrm{Kbp}$ windows. Topology

324 weightings for each window were then computed through determining the number of

325 unique subtrees that match each of the three possible topologies by iteratively sampling

326 a single haplotype from each species (Martin \& Van Belleghem, 2017). Windows were

327 discarded in topology weighting estimation if there were $<50$ SNPs and $<200$ SNPs

328 left from previous filtering steps for $10 \mathrm{Kbp}$ and $100 \mathrm{Kbp}$ windows, respectively.

Inference of incomplete lineage sorting

331 Because the speciation events that resulted in aspen species were close in time (see

332 Results), we expect the lineage sorting process relating these species to be incomplete.

333 Given the three aspen species and the outgroup poplar species (P. trichocarpa) with the

334 relationship as $(((P$. tremula, $P$. davidiana $), P$. tremuloides $), P$. trichocarpa $)$, we labeled

335 alleles in P. tremula, $P$. davidiana and P. tremuloies as A (ancestral allele) if they match

336 the reference allele of $P$. trichocarpa genome, and B (derived allele) otherwise. We then

337 considered segregating sites with $(((P$. tremula, P.davidiana $), P$. tremuloides $), P$.

338 trichocarpa) patterns as AABAs, ABAAs, BAAAs, ABBAs, BABAs and BBAAs. The

339 two SNP patterns ABBAs and BABAs can result from incomplete lineage sorting if we

340 assume no gene flow occurred among species (Durand, Patterson, Reich, \& Slatkin,

341 2011; Green et al., 2010). We calculated the level of incomplete lineage sorting (ILS) at

342 site $i$ in the genome as:

$344 \mathrm{ILS}=\left(C_{\mathrm{ABBA}(\mathrm{i})}+\mathrm{C}_{\mathrm{BABA}(\mathrm{i})}\right) / h$

345 where $h=\left(C_{\mathrm{BAAA}(\mathrm{i})}+C_{\mathrm{ABAA}(\mathrm{i})}+C_{\mathrm{AABA}(\mathrm{i})}+2\left(C_{\mathrm{BBAA}(\mathrm{i})}+C_{\mathrm{BABA}(\mathrm{i})}+C_{\mathrm{ABBA}(\mathrm{i})}\right) / 3\right.$ 
347 Because population samples were used for all species, at each site we used the

348 frequency of the derived allele in each species to effectively weight each segregating

349 site according to its fit to the six segregation patterns for the three aspen species

350 (Durand et al., 2011), with

351

$352 \quad C_{\mathrm{BAAA}(\mathrm{i})}=\hat{p}_{\mathrm{i} 1}\left(1-\hat{p}_{\mathrm{i} 2}\right)\left(1-\hat{p}_{\mathrm{i} 3}\right)\left(1-\hat{p}_{\mathrm{i} 4}\right)$

$353 \quad C_{\mathrm{ABAA}(\mathrm{i})}=\left(1-\hat{p}_{\mathrm{i} 1}\right) \hat{p}_{\mathrm{i} 2}\left(1-\hat{p}_{\mathrm{i} 3}\right)\left(1-\hat{p}_{\mathrm{i} 4}\right)$

$C_{\mathrm{AABA}(\mathrm{i})}=\left(1-\hat{p}_{\mathrm{i} 1}\right)\left(1-\hat{p}_{\mathrm{i} 2}\right) \hat{p}_{\mathrm{i} 3}\left(1-\hat{p}_{\mathrm{i} 4}\right)$

$C_{\mathrm{BBAA}(\mathrm{i})}=\hat{p}_{\mathrm{i} 1} \hat{p}_{\mathrm{i} 2}\left(1-\hat{p}_{\mathrm{i} 3}\right)\left(1-\hat{p}_{\mathrm{i} 4}\right)$

$356 \quad C_{\mathrm{BABA}(\mathrm{i})}=\hat{p}_{\mathrm{i} 1}\left(1-\hat{p}_{\mathrm{i} 2}\right) \hat{p}_{\mathrm{i} 3}\left(1-\hat{p}_{\mathrm{i} 4}\right)$

where $\hat{p}_{\mathrm{ij}}$ is the frequency of the derived allele at site $\mathrm{i}$ in species $\mathrm{j}$.

The calculation of ILS presents the counts of incomplete lineage sorting pattern

$362\left(C_{\mathrm{ABBA}(\mathrm{i})}\right.$ and $\left.\mathrm{C}_{\mathrm{BABA}(\mathrm{i})}\right)$ normalized by the total count of segregating sites $(h)$, which is a

363 proxy of the species tree topology height (Scally et al., 2012). We then summarized the

364 proportion of ILS over non-overlapping $10 \mathrm{Kbp}$ and $100 \mathrm{Kbp}$ windows or in bins with 365 varying distances from the nearest exon.

\subsection{Analyses of introgression}

368 We first tested for introgression between the three aspen species using the $D$-statistic, 369 also known as the ABBA-BABA tests. These tests evaluate the imbalance frequency of

370 site patterns (Durand et al., 2011; Green et al., 2010). Using P. trichocarpa as the 
371 outgroup, we expect equal counts of the two site patterns (ABBA and BABA) when

372 incomplete lineage sorting causes the site pattern discordance. If discordance is caused

373 by introgression, one of the site patterns is expected to be more prevalent than the other.

374 We applied two approaches to perform the $D$-statistic test. First, we used

375 -doAbbababa2 implemented in ANGSD v0.917 (Korneliussen et al., 2014) to directly

376 count $\mathrm{ABBA}$ and $\mathrm{BABA}$ sites and calculate the $D$ statistic without calling genotypes in

377 non-overlapping $10 \mathrm{Kbp}$ and $100 \mathrm{Kbp}$ windows for the whole genome. Then, jackknife

378 bootstrapping was conducted to estimate significance at the chromosome and

379 whole-genome level. Second, based on allele frequencies at each SNP called by GATK,

380 we calculated the $D$ statistic in $10 \mathrm{Kbp}$ and $100 \mathrm{Kbp}$ non-overlapping windows across

381 the genome with the python script ABBABABAwindows.py

382 (https://github.com/simonhmartin/genomics general) (Martin, Davey, \& Jiggins, 2014).

383 Following the detection of introgression among individuals at the genome level, we

384 used a modified $f$-statistic $\left(f_{\mathrm{d}}\right)$ (Martin et al., 2014) to estimate the proportion of

385 introgressed sites at the population level using ABBABABAwindows.py

386 (https://github.com/simonhmartin/genomics general) on non-overlapping $10 \mathrm{Kbp}$ and

$387100 \mathrm{Kbp}$ windows across the genome.

\subsection{Genome-wide scan for regions under positive and balancing selection in aspens}

390 To specifically test for the impact of positive and balancing selection on the genomic

391 landscape during speciation of aspens, we first used a composite likelihood ratio (CLR)

392 statistic implemented in SweepFinder2 (DeGiorgio, Huber, Hubisz, Hellmann, \&

393 Nielsen, 2016) to detect regions subject to recent positive selection or selective sweeps

394 in each of the three aspen species. The ancestral allelic state was defined by assuming

395 that the alleles that were the same as those found in the P. trichocarpa reference 
396 genome was the ancestral alleles. By contrasting the likelihood of the null hypothesis,

397 based on the unfolded site frequency spectrum (SFS) calculated across the genome

398 using $-\mathrm{f}$ option, with the likelihood of a model where the SFS has been altered by a

399 recent positive selection event, the CLR statistics was calculated in non-overlapping

400 windows of $10 \mathrm{Kbp}$. Within each species, windows with CLR values higher than the

$40199^{\text {th }}$ percentile of its distribution were identified as candidate region under selection.

402 Moreover, we identified regions under long-term balancing selection by estimating

403 the summary statistics, $\beta$ (beta score), which detects the clusters of variants with an

404 excess number of intermediate frequency polymorphisms (Siewert \& Voight, 2017).

405 Given that the signals of long-term balancing selection usually is localized to a narrow

406 genomic region (Gao, Przeworski, \& Sella, 2015), we used $1 \mathrm{Kbp}$ windows to calculate

$407 \beta$ values for each core SNPs in the three aspen species. We used the unfolded version of

$408 \beta$, with the ancestral and derived allelic states inferred based on comparisons with the

409 outgroup species $P$. trichocarpa. To prevent false positives, we filtered out SNPs with a

410 folded frequency lower than $20 \%$, and defined the SNPs with extreme $\beta$ scores in the

411 top $1 \%$ as significant. Furthermore, only SNPs that are significant in all three species

412 were considered as putative targets of long-term balancing selection. Finally, we binned

413 significant SNPs into $10 \mathrm{Kbp}$ windows for downstream comparisons.

414 Lastly, to assess the effects of positive and balancing selection on the genomic

415 architecture of speciation, we compared outlier windows that were identified as being

416 under positive or balancing selection with the remaining genomic regions using a

417 variety of population genetic statistics, including genetic diversity, divergence, lineage

418 sorting and introgression within and between the three closely related aspen species.

419 Differences between outlier windows and the genome-wide averages for all these

420 statistics were tested using Wilcoxon ranked-sum tests. To further examine whether any 
421 functional classes of genes were over-represented in these candidate regions, we

422 performed gene ontology (GO) analyses using the R package topGO 2.36.0 (Alexa \&

423 Rahnenführer 2009). Fisher's exact test was used to calculate the statistical significance

424 of enrichment, and GO terms with $P$-value lower than 0.01 were considered to be

425 significantly enriched.

\section{3. Results}

\subsection{Phylogenetic relationships, population structure and demographic history}

429 The genome alignment resulted in an average depth of $24.6 \times$ across all individuals

430 after quality control (Table S1). The PCA results revealed a clear distinction among the

431 four Populus species (Figure S4). Based on the Tracy-Widom test, only the first three

432 components were significant (Table S2). The first principal component (PC1; variance

433 explained $=28.79 \%$ ) separated $P$. trichocarpa from the three aspen species, while the

434 second component (PC2; variance explained=7.52\%) separated $P$. tremuloides from $P$.

435 tremula and P. davidiana. Finally, the third component (PC3, variance

436 explained $=5.33 \%$ ) separated $P$. tremula and $P$. davidiana. The clustering and genetic

437 relationships of the four species were also confirmed by the phylogenetic tree

438 constructed based on the entire chloroplast genomes (Figure 1B). Moreover, we

439 measured the number and length of shared IBD haplotypes within and between species

440 (Figure S5, S6; Table S3, S4). Compared to between-species comparisons, we found

441 much more extensively shared IBD haplotypes for within-species comparisons (Figure

442 S5), although haplotypes shared within P. tremuloides were shorter than the other three

443 species (Figure S6A; Table S4). This is likely owing to the higher recombination rate

444 and more rapid decay of linkage disequilibrium (LD) in $P$. tremuloides than other

445 species (Wang et al., 2016a). For the between-species comparisons, we did no observe 
446 any haplotype sharing between the three aspen species and P. trichocarpa, confirming

447 the distant relationship between aspens and poplars in the genus Populus (Figure S5,

448 Table S3). Within aspens, $P$. tremula and $P$. davidiana shared more and longer

449 haplotypes than either of them shared with P. tremuloides (Figure S5, S6B; Table S3,

450 S4), which also supports a closer relationship between these two species, as identified in

451 the chloroplast phylogeny.

452 To investigate the demographic and speciation histories of the four Populus species,

453 we first used MSMC to examine historical fluctuations in the effective population size

$454\left(N_{\mathrm{e}}\right)$ for each species. The results showed that all species experienced a period of

455 population decline during the early Pleistocene cooling (2.5-0.9 million years ago, Mya)

456 (Figure 1C). Compared with the three aspen species, P. trichocarpa experienced a more

457 dramatic population decline during this period (Figure 1C, Figure S7), which likely

458 explain the much lower genetic diversity observed in this species relative to others

459 (Table S5). The two North American species, $P$. tremuloides and $P$. trichocarpa,

460 experienced a population expansion from the start of the last ice age (110 thousand

461 years ago, Kya) until the last glacial maximum (LGM, 23-18 Kya) whereas the

462 European species $P$. tremula remained relatively stable. On the other hand, the eastern

463 Asian species, P. davidiana, showed pattern of population decline during the entire

464 period (Figure 1C, Figure S7). Our results therefore revealed that before the LGM,

465 forest trees distributed in different continents experienced asynchronous demographic

466 responses to Pleistocene climate changes (Bai et al., 2018). During and following the

467 LGM, all four species experienced a population decline followed by a subsequent rapid

468 population expansion (Figure 1C).

469 Because of the distant phylogenetic relationship and low levels of shared 470 polymorphism between P. trichocarpa and the three aspen species (Wang et al., 2016a), 
471 we therefore explicitly focused on inferring the demographic parameters of the

472 speciation history for the three aspens. After evaluating a total of twenty-nine models

473 (Figure S8), the best-supported model (Figure S8; Table S6) suggests that the Eurasian

474 lineage (the common ancestor of $P$. tremula and $P$. davidiana) split from the North

475 American lineage (P. tremuloides) at 2.4 Mya (bootstrap range [BP]: 2.1-3.2 Mya),

476 which is in accordance with our earlier estimates on the divergence time between $P$.

477 tremula and P. tremuloides (Wang, Street, Scofield, \& Ingvarsson, 2016b). The

478 European lineage ( $P$. tremula) and the East-Asian lineage ( $P$. davidiana) diverged $\sim 1.7$

479 Mya (BP: 1.5-2.1 Mya) (Figure 1D, Table S7). Our results detected low levels of 480 ancient gene flow between $P$. tremula and $P$. davidiana, and between $P$. tremula and $P$. 481 tremuloides following speciation until around 847 Kya (BP: 539Kya-1.0 Mya) (Figure 482 1D). After this period the species have remained isolated which is also reflected by their 483 current disjunct geographic distributions (Figure 1A). Compared to the Eurasian lineage 484 of aspens, $P$. tremuloides has experienced a notable population expansion in the recent 485 past ( 772 Kya, BP: 440-887 Kya), which is consistent with its genome-wide excess of 486 rare alleles (Figure S2).

\subsection{General patterns of genome-wide diversity and differentiation}

489 We further characterized genome-wide patterns of nucleotide diversity $(\pi)$, 490 population recombination rate $(\rho)$ and divergence $\left(F_{\mathrm{ST}}\right.$ and $\left.\mathrm{d}_{\mathrm{xy}}\right)$ for the four Populus 491 species (Figure 2A; Table S5, Table S8-S10). At the species level, $\pi$ varied markedly 492 between species, ranging from 0.0063 in $P$. trichocarpa to 0.0148 in $P$. tremuloides, but 493 the average genomic diversity was very similar across the three aspen species (Table

494 S5). In contrast to the patterns observed for $\pi$, the population-scaled recombination rate, $495 \rho$, was much higher in $P$. tremuloides $\left(0.0273 \mathrm{bp}^{-1}\right)$ than in the other three species 
$496\left(0.0096 \mathrm{bp}^{-1}-0.0139 \mathrm{bp}^{-1}\right)$ (Table S8). Variation in genetic divergence $\left(F_{\mathrm{ST}}\right.$ and $\left.\mathrm{d}_{\mathrm{xy}}\right)$

497 among the six species pairs reveals the continuous nature of differentiation along the

498 speciation continuum, with $P$. tremula and $P$. davidiana showing the lowest levels of

499 divergence and with the highest divergence observed between aspens and $P$.

500 trichocarpa (Figure 2A, Table S9, S10).

501 At the genome level, patterns of genetic diversity and divergence show high levels

502 of parallelism in all pairwise comparisons. The genome-wide profiles of $\pi$ (average

503 Spearman's $\rho=0.71$ ) and $\rho$ (average Spearman's $\rho=0.18$ ) were positively correlated in

504 all possible species pairs (Figure 2B, Table S11). We found little evidence for an

505 association between either $\pi$ or $\rho$ and the local mutation rate $(\mu$, approximated by the

506 four-fold synonymous substitution rate) (Figure 2B, Table S11). Hence, the broad-scale

507 variation in genetic diversity is conserved across the diverging lineages, which likely

508 arise from a common genomic architecture where linked selection has played a major

509 role in shaping local genomic diversity (Burri, 2017). This is further highlighted by the

510 conserved landscape of recombination rate variation across the genomes of the species

511 and the strong degree of genome synteny that we observed between the genomes of

512 aspens and poplars (Lin et al., 2018). Second, we found that the differentiation

513 landscapes were highly correlated among all species pairs both for the relative $\left(F_{\mathrm{ST}}\right)$ and

514 the absolute $\left(\mathrm{d}_{\mathrm{xy}}\right)$ measures of genetic differentiation (Figure 2B, Figure S9, Table S11).

515 The highly similar landscape of differentiation among different species pairs could

516 imply phylogenetically conserved genomic features, e.g. conserved landscapes of

517 functional densities and recombination (Burri, 2017; Vijay et al., 2017). Moreover,

518 significantly negative correlations between $F_{\mathrm{ST}}$ and $\pi$ were found in all pairwise

519 comparisons (Figure 2B, Figure S9, Table S11), which is in line with the observation

520 that $F_{\mathrm{ST}}$ is sensitive to intra-specific nucleotide diversity (Charlesworth, 1998). In 
521 contrast, only weak correlations were observed between $d_{x y}$ and $\pi$. Because $d_{x y}$ largely

522 reflects diversity in a common ancestor (Cruickshank \& Hahn, 2014), a weak

523 correlation between $\mathrm{d}_{\mathrm{xy}}$ and $\pi$ implies that ancestral diversity might have little impact on

524 extant diversity in the different Populus species. In addition to extant diversity, $\mathrm{d}_{\mathrm{xy}}$ was

525 only weakly correlated with $F_{\mathrm{ST}}$ across all comparisons (Figure 3B, Figure S9, Table

526 S11), which further implies that ancestral polymorphisms have had limited contribution

527 to the genomic divergence of extant species (Cruickshank \& Hahn, 2014).

\subsection{Topology weighting reveals phylogenetic discordance and ancient introgression}

531 Even if the analyses of current population structure and genomic divergence support a 532 clear species relationship for the four Populus species, (((P. tremula, P. davidiana), $P$.

533 tremuloides), P. trichocarpa), we used a topology weighting approach to explore to

534 what degree the 'species tree' was congruent across the entire genome. Using $P$.

535 trichocarpa as an outgroup, our results reveal widespread incongruence in local

536 genealogies in either $10 \mathrm{Kbp}$ or $100 \mathrm{Kbp}$ non-overlapping windows across the genome

537 (Figure 3, Figure S10). The most prevalent topology, ((P. tremula, $P$. davidiana), $P$.

538 tremuloides), which reflects the likely 'species topology', has an average weighting of

$53954.7 \%$ and $76.5 \%$ across the genome in $10 \mathrm{Kbp}$ and $100 \mathrm{Kbp}$ windows, respectively. Of

540 the other two topologies, the ((P. tremula, $P$. tremuloides $),$ P. davidiana) topology was

541 much more common (27.0\% and $17.6 \%$ for $10 \mathrm{Kbp}$ and $100 \mathrm{Kbp}$ windows) compared

542 to ((P. davidiana, P. tremuloides), P. tremula) $(18.3 \%$ and $5.9 \%$ for $10 \mathrm{Kbp}$ and 100

543 Kbp windows) (Figure 3, Table S12). In general, we observed that larger windows (100

$544 \mathrm{Kbp}$ ) produced higher rates of monophyly (windows with a weighting of 1) and a 
545 greater fraction of resolved trees compared to the smaller windows (10 Kbp) (Figure

546 S10, Table S13).

547 Interestingly, in contrast to all other chromosomes where all three topologies were

548 observed, chromosome 19, which is known to harbor the sex determination region in

549 Populus (Yin et al., 2008), showed only a single monophyletic grouping of the 'species

550 topology' (Figure 3). Such a pattern is consistent with the expectation that lineage

551 sorting is faster on sex chromosomes compared to autosomes because of its smaller

552 effective population size (Meisel \& Connallon, 2013; Vicoso \& Charlesworth, 2006).

553 Overall, both incomplete lineage sorting (ILS) and introgression can result in

554 discordance between the local topology and the species tree for recently diverged

555 species. Given that ILS is expected to generate equal frequencies of the alternative

556 topologies (Durand et al., 2011; Mailund et al., 2014), the more frequent topology of

557 ((P. tremula, P. tremuloides $), P$. davidiana) is likely explained by the occurrence of

558 introgression between $P$. tremula and $P$. tremuloides. We therefore compared the

559 distribution of the branch lengths separating each pair of aspen species among all

560 topology types. Compared to the expectation that species with recent introgression tend

561 to be separated by short branches (Fontaine et al., 2015; Martin \& Van Belleghem,

562 2017), the branch distances between $P$. tremula and P. tremuloides were not obviously

563 different from other species-pairs across topology comparisons (Figure S11). This

564 pattern is most likely caused by ancient hybridization between these two species where

565 genetic drift has eradicated most signatures of gene flow after an ancient introgression

566 event (Schumer, Cui, Powell, Rosenthal, \& Andolfatto, 2016).

567 To further investigate patterns of ancient introgression between $P$. tremula and $P$.

568 tremuloides, we calculated two statistics associated with the ABBA-BABA test across

569 the genome. The $D$-statistic is used to test for ancient gene flow by comparing the 
570 imbalance of ABBAs and BABAs, and the $f_{\mathrm{d}}$-statistic is used to estimate the fraction of

571 the genome shared through ancient introgression. For the $D$-statistics, we also

572 implemented two different approaches, which differed in whether the called genotypes

573 was relied or not. We find that the estimates of the two approaches are highly correlated

574 with each other (Figure S12), suggesting that this statistic is robust to identify

575 introgression regardless of which type of data is used. Genome-wide estimates of the

$576 D$-statistic and $f$-statistic showed a general pattern of positive values over $10 \mathrm{Kbp}$ and

$577100 \mathrm{Kbp}$ non-overlapping windows (Table S14), confirming that $P$. tremuloides has a

578 closer genetic relationship with $P$. tremula than with $P$. davidiana. Thus, the significant

579 asymmetry in genetic relationship together with the excess of shared sequence

580 polymorphism between $P$. tremula and $P$. tremuloides (Figure S13) all provide evidence

581 for historical gene flow between the currently allopatric Eurasian and North American

582 aspen species.

3.4 Long-term effects of selection in shaping patterns of diversity, divergence, incomplete lineage sorting (ILS) and levels of introgression in Populus species

586 To evaluate the impact of natural selection on genetic diversity, divergence, ILS and

587 gene flow in the context of speciation, we examined the correlations between these 588 genetic parameters and factors affecting the extent and efficiency of selection. First, 589 regions with a high density of potential targets for selection are expected to experience 590 stronger linked selection simply because selection occurs more often in such regions 591 (Al-Shahrour et al., 2010; Flowers et al., 2011). We therefore examined the relationship 592 between intraspecific diversity, species divergence and the density of functional 593 elements, defined as the proportion of protein-coding sites within a $10 \mathrm{Kbp}$ or $100 \mathrm{Kbp}$ 594 window (coding density). We hypothesized that if selection contributes to the reduction 
595 of diversity at linked neutral sites, its effect is expected to be more pronounced in

596 regions with greater content of functional elements (Ravinet et al., 2017). Consistent

597 with this prediction, we observed a significantly negative relationship between $\pi$ and

598 functional content (Figure 4A). This correlation was robust to the presence of 599 confounding factors such as GC content, recombination rate and the choice of window 600 size (Table S15).

601 Moreover, if natural selection was acting on the ancestral polymorphisms prior to 602 the divergence of the two descendant lineages, it could also have an effect on the 603 genetic divergence between species (Munch et al., 2016; Scally et al., 2012). We

604 therefore examined the relationship between interspecies divergence (both $F_{\mathrm{ST}}$ and $\mathrm{d}_{\mathrm{xy}}$ ) 605 and coding density, and observed negative relationships for both $F_{\mathrm{ST}}$ and $\mathrm{d}_{\mathrm{xy}}$ (Figure 606 4B, C), especially between species with longer divergence times (e.g. aspens and $P$. 607 trichocarpa) (Table S17, Table S19). Indeed, if a region experiences natural selection 608 during divergence, it should show lower $\pi$ within species and higher $F_{\text {ST }}$ between 609 species because $F_{\mathrm{ST}}$ is sensitive to intra-specific genetic variation (Cruickshank \& 610 Hahn, 2014). Accordingly, a positive correlation between coding density and $F_{\mathrm{ST}}$ is 611 predicted. The opposite pattern we observe here indicates that long-term natural 612 selection, most likely due to background selection in functional regions, has 613 continuously contributed to the reduced ancestral polymorphism and genetic divergence 614 in regions with greater functional content (Phung et al., 2016). In fact, because of the 615 accumulation of the large amount of new mutations since speciation, ancestral 616 polymorphism may only account for a small amount of the overall average divergence 617 between distantly related species (Edwards \& Beerli, 2000). However, the variance of 618 ancestral polymorphism, largely affected by natural selection in ancestral populations, 619 can on the other hand make a substantial contribution to the variability of genome-wide 
620 patterns of divergence between species (McVicker, Gordon, Davis, \& Green, 2009;

621 Phung et al., 2016).

622 To further explore the role of natural selection during the divergence of the three 623 aspen species, we examined the extent of ILS across the genome, which can aid to infer 624 evolutionary process in ancestral populations (Mailund et al., 2014; Pease \& Hahn, 625 2013). The pattern of ILS along the genome offers information about the local 626 differences in the ancestral effective population size of the aspen ancestor (Pamilo \& 627 Nei, 1988). Both purifying and positive selection in the ancestral population are 628 expected to reduce ancestral population size in regions targeted by selection, resulting in 629 increased rates for lineages to coalesce and leaving less available for ILS (Dutheil, 630 Munch, Nam, Mailund, \& Schierup, 2015; Munch et al., 2016; Prüfer et al., 2012; 631 Scally et al., 2012). In agreement with this, we found that the fraction of ILS decreases 632 with increasing coding density (Figure 4D), and this relationship remained even after 633 correcting for the confounding variables (Table S21). Within coding exons, ILS is 19\%

634 lower and the suppression of ILS extends several thousand bps away from coding genes 635 (Figure S14). Similarly, the proportion of the topology reflecting the true species tree 636 increases with coding density (Figure 4E; Table S21).

637 In addition, given that the level of admixture estimated by $f_{\mathrm{d}}$ between $P$. tremula 638 and $P$. tremuloides show considerable heterogeneity across the genome (Figure S15), 639 we examined whether selection may have played a role in shaping genome-wide 640 patterns of introgression. We estimated the relationship between $f_{\mathrm{d}}$ and coding density 641 and found a significantly negative correlation (Figure 4F; Table S21), indicating that 642 there is greater selection against introgressed alleles in regions enriched for genes 643 (Harris \& Nielsen, 2016). The occurrence of this pattern is not likely an artefact of 644 reduced power, as regions with a high density of functionally important elements are 
645 expected to have experienced stronger long-term selection and exhibit lower levels of

646 ILS. Accordingly, our power to detect introgression is expected to be elevated close to

647 these regions (Sankararaman et al., 2014; Sankararaman, Mallick, Patterson, \& Reich,

648 2016). Taken together, it is clear that natural selection has had a strong impact on

649 patterns of phylogenetic discordance across the genome among closely related aspen

650 species. However, it is not yet clear to what extent this heterogeneity might be due to

651 incomplete lineage sorting of ancestral polymorphisms or due to ancient introgression.

652 More explicit experimental designs in future studies are needed to tease apart these

653 different processes and explore how natural selection and hybridization act in

654 combination to shape the genome-wide phylogenetic heterogeneity among recently

655 diverged species.

656 In addition to the local density of functional elements, recombination rates can also

657 interact with natural selection to influence the genomic distribution of genetic diversity

658 and divergence (Figure 4). High recombination can rapidly decouple linked loci and

659 restrict the effect of selection on linked neutral sites (Begun \& Aquadro, 1992; Cutter \&

660 Payseur, 2013). We found that $\pi$ and $F_{\mathrm{ST}}$ showed positive and negative correlations,

661 respectively, with local recombination rates (Figure 4A-C; Table S16, S18, S20). In

662 contrast to the predicted pattern of speciation with gene flow where reduced $d_{x y}$ is

663 expected in regions of high recombination (Nachman \& Payseur, 2012), we did not find

664 any relationship between $\mathrm{d}_{\mathrm{xy}}$ and recombination rate. These observations are in

665 accordance with the expectation that linked selection is prevalent and has genome-wide

666 effects in shaping patterns of genetic diversity and divergence at linked sites in Populus

667 (Nachman \& Payseur, 2012; Wang et al., 2016b). On the other hand, we found that the

668 incidence of ILS increases with the recombination rate (Figure 4D), which was still

669 observable even after correcting for the confounding variables of coding density and 
670 GC content (Table S22). Given that variation in ILS across the genome approximately

671 reflects variation in ancestral $N_{\mathrm{e}}$ (Degnan \& Salter, 2005; Pamilo \& Nei, 1988), the

672 stronger effects of recurrent natural selection in low-recombination regions also reduced

$673 N_{\mathrm{e}}$ in ancestral populations and hence made ILS less likely to occur (Charlesworth et al.,

674 1993; Martin, Davey, Salazar, \& Jiggins, 2019; Pease \& Hahn, 2013). We did not find

675 obvious correlation between $f_{\mathrm{d}}$ and recombination rate (Figure $4 \mathrm{~F}$, Table S22), might

676 because barriers to introgression has been sculpted by long-term selection and genetic

677 drift after the ancient gene flow and cannot be predicted by recombination rate

678 estimated from current populations. Overall, all these results suggest that the patterns of

679 diversity, divergence and genealogical relationships among the three closely related

680 aspen species are not randomly distributed along the genome, but instead are strongly

681 structured by the interaction between widespread natural selection and intrinsic genomic

682 features, as well as their influence on retention of signatures of ancient gene flow.

6843.5 The impact of positive and balancing selection on genomic architecture of $685 \quad$ speciation

686 Although widespread background selection is likely to have had a large effect in 687 shaping the heterogeneous genomic landscape of variation within and between species 688 (Burri, 2017; Charlesworth, 2012), we were interested in assessing whether positive 689 selection or long-term balancing selection have also played important roles in driving 690 these processes. To identify the impact of positive selection, we performed a 691 composite-likelihood based (CLR) test to scan the genomes for signals of positive 692 selection in each of the three aspen species (Figure 5A). For each species, we 693 considered the windows with a CLR value in the top 1 percentile as candidate region 694 under positive selection. In total, we detected 538 outlier windows across the three 
695 species, and only 13 among them were shared by all species (Figure 5B). Our results

696 suggest that most putative sweeps are likely species-specific and may result from

697 relatively recent positive selection that has occurred independently in various lineages

698 after speciation. Compared to genome-wide averages, outlier windows have

699 significantly lower nucleotide diversity, lower recombination rates, higher $F_{\mathrm{ST}}$ but

700 similar $d_{x y}$ (Figure 5C). In addition, the outlier windows show significantly higher

701 average weightings of the 'species topology' (Topo2) and lower levels of ILS compared

702 to genomic background (Figure 5C,D). The ancestral admixture proportion $\left(f_{\mathrm{d}}\right)$ between

$703 \quad P$. tremula and $P$. tremuloides is also significantly reduced in the outlier windows

704 (Figure 5D), suggesting that strong selection in these regions may have contributed to

705 the reproductive barriers isolating closely related species (Martin et al., 2019).

706 To further study how long-term balancing selection may have driven the evolution

707 of the genomic landscape during speciation, we used a summary statistics, $\beta$ (beta score),

708 to search for signals of balancing selection across the genome for each aspen species

709 (Siewert \& Voight, 2017). As we did for positive selection, we only consider variants

710 with $\beta$ scores falling in the top $1 \%$ as candidate variants. Furthermore, variants

711 simultaneously detected in all three species are considered as potential targets of

712 long-term balancing selection. With this criteria we identified a total of 519 variants

713 putatively under long-term balancing selection across the three aspen species (Figure

714 6A,B). These variants were unevenly distributed in the genome, and to make them

715 comparable to our previous analyses we clustered them into 32 regions of $10 \mathrm{Kbp}$

716 windows (Figure 6A). We found significantly higher nucleotide diversity, higher

717 recombination rates, lower $F_{\mathrm{ST}}$, and higher $\mathrm{d}_{\mathrm{xy}}$ in the regions under balancing selection

718 compared to the genomic background (Figure 6C). Moreover, we found lower

719 weightings of the 'species topology', higher ILS, and lower $f_{\mathrm{d}}$ in the candidate balancing 
720 selection regions although the results were not significant, likely due to the small

721 number of windows showing evidence for balancing selection (Figure 6C,D). We

722 therefore infer that long-term balancing selection may not only influence the genomic

723 landscape of diversity and divergence but may also play a role in shaping the

724 genealogical relationship and barriers to introgression among closely related species

725 (Charlesworth, 2006; Wang et al., 2019).

726 Finally, to assess whether there were any specific biological functions that were

727 significantly over-represented on genes located in regions identified as undergoing

728 either positive (506 genes) or long-term balancing selection (32 genes), we performed

729 gene ontology (GO) enrichment analysis. We did not detect over-representation for any

730 functional category among the candidate genes under long-term balancing selection. In

731 contrast, we identified 31 significantly enriched GO categories (Fisher's exact test,

$732 P<0.01$ ) for genes under positive selection (Table S23). These GO clusters were

733 primarily associated with metabolic processes (DNA, nucleic acid, cellular

734 macromolecule and aromatic compound, molybdopterin confactor), biosynthetic

735 processes (molybdopterin confactor, vitamin B6), cell morphogenesis and gene

736 expression regulation. Together these functional clusters are biologically relevant for

737 plant adaptation, because the biosynthesis of a panoply of diverse natural chemicals

738 serve as important adaptive strategies for sessile long-lived trees to adapt to

739 ever-changing abiotic and biotic environments (Weng, 2014).

741 4. Discussion

742 Much of our knowledge of how genomic landscape builds in the speciation process is

743 drawn from studies focusing on two young species pairs with ongoing gene flow. Very

744 few examples of now-allopatric species pairs along the speciation continuum have been 
745 investigated. Here, we focus our research on four widespread Populus species that are

746 allopatrically distributed in northern Hemisphere. After characterizing their speciation

747 and demographic histories, we find that species in different continent exhibited

748 idiosyncratic responses to Pleistocene climate changes. In addition, ancient gene flow

749 was detected between extant Eurasian and North American aspen species (P. tremula

750 and $P$. tremuloides). We also investigated the evolutionary forces that have shaped

751 genome-wide patterns of variations within and between species. Our results have found

752 substantial variation in genetic diversity, divergence, species relationships and the

753 extent of introgression along the genome. Variation in these patterns is predictable and

754 can be largely explained by genome-wide variation in the strength and extent of both

755 recent and ancient selection, which depends on the recombination rate and the local

756 density of functional sites. Our findings therefore provide evidence of how recurrent

757 selection interacts with genomic features to shape the genomic landscape during species

758 divergence. We further demonstrate that not only background selection, positive and

759 long-term balancing selection also play crucial roles in shaping genomic variation and

760 phylogenetic relationship among the recently diverged aspen species. Overall, this study

761 highlights the striking impacts of natural selection in shaping within- and between-

762 species genomic variation through speciation.

\section{Acknowlegements}

765 All analyses were performed on resources provided by the Swedish National

766 Infrastructure for Computing (SNIC) at Uppsala Multidisciplinary Center for Advanced

767 Computational Science (UPPMAX ) under the projects SNIC2016-7-89 and SNIC

768 2017/1-499. Financial support was provided by National Natural Science Foundation of

769 China (31971567) and the Fundamental Research Funds for the Central Universities. 


\section{References}

Abbott, R., Albach, D., Ansell, S., Arntzen, J. W., Baird, S. J., Bierne, N., . . Buggs, R. (2013). Hybridization and speciation. Journal of Evolutionary Biology, 26(2), 229-246.

Al-Shahrour, F., Minguez, P., Marqués-Bonet, T., Gazave, E., Navarro, A., \& Dopazo, J. (2010). Selection upon genome architecture: conservation of functional neighborhoods with changing genes. PLoS Computational Biology, 6(10), e1000953.

Alexa, A., \& Rahnenführer, J. (2009). Gene set enrichment analysis with topGO. Bioconductor Improv, 27.

Bai, W. N., Yan, P. C., Zhang, B. W., Woeste, K. E., Lin, K., \& Zhang, D. Y. (2018). Demographically idiosyncratic responses to climate change and rapid Pleistocene diversification of the walnut genus Juglans (Juglandaceae) revealed by whole-genome sequences. New Phytologist, 217(4), 1726-1736.

Begun, D. J., \& Aquadro, C. F. (1992). Levels of naturally occurring DNA polymorphism correlate with recombination rates in D. melanogaster. Nature, 356(6369), 519.

Browning, B. L., \& Browning, S. R. (2009). A unified approach to genotype imputation and haplotype-phase inference for large data sets of trios and unrelated individuals. The American Journal of Human Genetics, 84(2), 210-223.

Browning, B. L., \& Browning, S. R. (2013). Improving the accuracy and efficiency of identity-by-descent detection in population data. Genetics, 194(2), 459-471.

Burri, R. (2017). Interpreting differentiation landscapes in the light of long-term linked selection. Evolution Letters, 1(3), 118-131.

Burri, R., Nater, A., Kawakami, T., Mugal, C. F., Olason, P. I., Smeds, L., . . . Garamszegi, L. Z. (2015). Linked selection and recombination rate variation drive the evolution of the genomic landscape of differentiation across the speciation continuum of Ficedula flycatchers. Genome Research, 25(11), 1656-1665.

Chan, A. H., Jenkins, P. A., \& Song, Y. S. (2012). Genome-wide fine-scale recombination rate variation in Drosophila melanogaster. PLoS Genetics, 8(12), e1003090.

Charlesworth, B. (1998). Measures of divergence between populations and the effect of forces that reduce variability. Molecular Biology and Evolution, 15(5), 538-543.

Charlesworth, B. (2012). The effects of deleterious mutations on evolution at linked sites. Genetics, 190(1), 5-22.

Charlesworth, B., Morgan, M., \& Charlesworth, D. (1993). The effect of deleterious mutations on neutral molecular variation. Genetics, 134(4), 1289-1303.

Charlesworth, D. (2006). Balancing selection and its effects on sequences in nearby genome regions. PLoS Genetics, 2(4), e64.

Comeron, J. M., Williford, A., \& Kliman, R. (2008). The Hill-Robertson effect: evolutionary consequences of weak selection and linkage in finite populations. Heredity, 100(1), 19.

Cruickshank, T. E., \& Hahn, M. W. (2014). Reanalysis suggests that genomic islands of speciation are due to reduced diversity, not reduced gene flow. Molecular Ecology, 23(13), 3133-3157. 
Cutter, A. D., \& Payseur, B. A. (2013). Genomic signatures of selection at linked sites: unifying the disparity among species. Nature Reviews Genetics, 14(4), 262.

Danecek, P., Auton, A., Abecasis, G., Albers, C. A., Banks, E., DePristo, M. A., . . . Sherry, S. T. (2011). The variant call format and VCFtools. Bioinformatics, 27(15), 2156-2158.

DeGiorgio, M., Huber, C. D., Hubisz, M. J., Hellmann, I., \& Nielsen, R. (2016). SweepFinder2: increased sensitivity, robustness and flexibility. Bioinformatics, 32(12), 1895-1897.

Degnan, J. H., \& Salter, L. A. (2005). Gene tree distributions under the coalescent process. Evolution, 59(1), 24-37.

Delmore, K. E., Lugo Ramos, J. S., Van Doren, B. M., Lundberg, M., Bensch, S., Irwin, D. E., \& Liedvogel, M. (2018). Comparative analysis examining patterns of genomic differentiation across multiple episodes of population divergence in birds. Evolution Letters, 2(2), 76-87.

DePristo, M. A., Banks, E., Poplin, R., Garimella, K. V., Maguire, J. R., Hartl, C., . . Hanna, M. (2011). A framework for variation discovery and genotyping using next-generation DNA sequencing data. Nature Genetics, 43(5), 491.

Du, S., Wang, Z., Ingvarsson, P. K., Wang, D., Wang, J., Wu, Z., . . Zhang, J. (2015). Multilocus analysis of nucleotide variation and speciation in three closely related P opulus (S alicaceae) species. Molecular Ecology, 24(19), 4994-5005.

Durand, E. Y., Patterson, N., Reich, D., \& Slatkin, M. (2011). Testing for ancient admixture between closely related populations. Molecular Biology and Evolution, 28(8), 2239-2252.

Dutheil, J. Y., Munch, K., Nam, K., Mailund, T., \& Schierup, M. H. (2015). Strong selective sweeps on the $\mathrm{X}$ chromosome in the human-chimpanzee ancestor explain its low divergence. PLoS Genetics, 11(8), e1005451.

Eckenwalder, J. E. (1996). Systematics and evolution of Populus. Biology of Populus and its Implications for Management and Conservation, 7, 32.

Edwards, S., \& Beerli, P. (2000). Perspective: gene divergence, population divergence, and the variance in coalescence time in phylogeographic studies. Evolution, 54(6), 1839-1854.

Ellegren, H., \& Galtier, N. (2016). Determinants of genetic diversity. Nature Reviews Genetics, 17(7), 422.

Excoffier, L., Dupanloup, I., Huerta-Sánchez, E., Sousa, V. C., \& Foll, M. (2013). Robust demographic inference from genomic and SNP data. PLoS Genetics, 9(10), e1003905.

Feder, J. L., Egan, S. P., \& Nosil, P. (2012). The genomics of speciation-with-gene-flow. Trends in Genetics, 28(7), 342-350.

Flaxman, S. M., Wacholder, A. C., Feder, J. L., \& Nosil, P. (2014). Theoretical models of the influence of genomic architecture on the dynamics of speciation. Molecular Ecology, 23(16), 4074-4088.

Flowers, J. M., Molina, J., Rubinstein, S., Huang, P., Schaal, B. A., \& Purugganan, M. D. (2011). Natural selection in gene-dense regions shapes the genomic pattern of polymorphism in wild and domesticated rice. Molecular Biology and Evolution, 29(2), 675-687.

Fontaine, M. C., Pease, J. B., Steele, A., Waterhouse, R. M., Neafsey, D. E., Sharakhov, I. V., . . Kakani, E. (2015). Extensive introgression in a malaria vector species complex revealed by phylogenomics. Science, 347(6217), 1258524. 
866

867

868

869

870

871

872

873

874

875

876

877

878

879

880

881

882

883

884

885

886

887

888

889

890

891

892

893

894

895

896

897

898

899

900

901

902

903

904

905

906

907

908

909

910

911

912

Fumagalli, M., Vieira, F. G., Korneliussen, T. S., Linderoth, T., Huerta-Sánchez, E., Albrechtsen, A., \& Nielsen, R. (2013). Quantifying population genetic differentiation from next-generation sequencing data. Genetics, 195(3), 979-992.

Fumagalli, M., Vieira, F. G., Linderoth, T., \& Nielsen, R. (2014). ngsTools: methods for population genetics analyses from next-generation sequencing data. Bioinformatics, 30(10), 1486-1487.

Gao, Z., Przeworski, M., \& Sella, G. (2015). Footprints of ancient-balanced polymorphisms in genetic variation data from closely related species. Evolution, 69(2), 431-446.

Green, R. E., Krause, J., Briggs, A. W., Maricic, T., Stenzel, U., Kircher, M., . . . Fritz, M. H.-Y. (2010). A draft sequence of the Neandertal genome. Science, 328(5979), 710-722.

Guerrero, R. F., \& Hahn, M. W. (2017). Speciation as a sieve for ancestral polymorphism. Molecular Ecology, 26(20), 5362-5368.

Hamzeh, M., \& Dayanandan, S. (2004). Phylogeny of Populus (Salicaceae) based on nucleotide sequences of chloroplast TRNT-TRNF region and nuclear rDNA. American Journal of Botany, 91(9), 1398-1408.

Han, F., Lamichhaney, S., Grant, B. R., Grant, P. R., Andersson, L., \& Webster, M. T. (2017). Gene flow, ancient polymorphism, and ecological adaptation shape the genomic landscape of divergence among Darwin's finches. Genome Research, 27(6), 1004-1015.

Harris, K., \& Nielsen, R. (2016). The genetic cost of Neanderthal introgression. Genetics, 203(2), 881-891.

Hart, J., De Araujo, F., Thomas, B., \& Mansfield, S. (2013). Wood quality and growth characterization across intra-and inter-specific hybrid aspen clones. Forests, 4(4), 786-807.

Hobolth, A., Dutheil, J. Y., Hawks, J., Schierup, M. H., \& Mailund, T. (2011). Incomplete lineage sorting patterns among human, chimpanzee, and orangutan suggest recent orangutan speciation and widespread selection. Genome Research, 21(3), 349-356.

Hudson, R. R., \& Kaplan, N. L. (1995). Deleterious background selection with recombination. Genetics, 141(4), 1605-1617.

Hurst, L. D., Pál, C., \& Lercher, M. J. (2004). The evolutionary dynamics of eukaryotic gene order. Nature Reviews Genetics, 5(4), 299.

Jansson, S., \& Douglas, C. J. (2007). Populus: a model system for plant biology. Annual Review of Plant Biology, 58, 435-458.

Kaplan, N. L., Hudson, R., \& Langley, C. (1989). The" hitchhiking effect" revisited. Genetics, 123(4), 887-899.

Koch, M. A., Haubold, B., \& Mitchell-Olds, T. (2000). Comparative evolutionary analysis of chalcone synthase and alcohol dehydrogenase loci in Arabidopsis, Arabis, and related genera (Brassicaceae). Molecular Biology and Evolution, 17(10), 1483-1498.

Korneliussen, T. S., Albrechtsen, A., \& Nielsen, R. (2014). ANGSD: analysis of next generation sequencing data. BMC Bioinformatics, 15(1), 356.

Lamichhaney, S., Berglund, J., Almén, M. S., Maqbool, K., Grabherr, M., Martinez-Barrio, A., . . . Zamani, N. (2015). Evolution of Darwin's finches and their beaks revealed by genome sequencing. Nature, 518(7539), 371. 
Lee, T.-H., Guo, H., Wang, X., Kim, C., \& Paterson, A. H. (2014). SNPhylo: a pipeline to construct a phylogenetic tree from huge SNP data. BMC Genomics, 15(1), 162.

Li, H. (2013). Aligning sequence reads, clone sequences and assembly contigs with BWA-MEM. arXiv:1303.3997.

Li, H., \& Durbin, R. (2009). Fast and accurate short read alignment with Burrows-Wheeler transform. Bioinformatics, 25(14), 1754-1760.

Li, H., Handsaker, B., Wysoker, A., Fennell, T., Ruan, J., Homer, N., . . . Durbin, R. (2009). The sequence alignment/map format and SAMtools. B ioinformatics, 25(16), 2078-2079.

Lin, Y.-C., Wang, J., Delhomme, N., Schiffthaler, B., Sundström, G., Zuccolo, A., . . . Cossu, R. M. (2018). Functional and evolutionary genomic inferences in Populus through genome and population sequencing of American and European aspen. Proceedings of the National Academy of Sciences, 115(46), E10970-E10978.

Lohmueller, K. E., Albrechtsen, A., Li, Y., Kim, S. Y., Korneliussen, T., Vinckenbosch, N., . . Grarup, N. (2011). Natural selection affects multiple aspects of genetic variation at putatively neutral sites across the human genome. PLoS Genetics, 7(10), e1002326.

Lohse, M., Bolger, A. M., Nagel, A., Fernie, A. R., Lunn, J. E., Stitt, M., \& Usadel, B. (2012). R obi NA: A user-friendly, integrated software solution for RNA-Seq-based transcriptomics. Nucleic Acids Research, 40(W1), W622-W627.

Ma, T., Wang, K., Hu, Q., Xi, Z., Wan, D., Wang, Q., . . Abbott, R. J. (2018). Ancient polymorphisms and divergence hitchhiking contribute to genomic islands of divergence within a poplar species complex. Proceedings of the National Academy of Sciences, 115(2), E236-E243.

Mailund, T., Munch, K., \& Schierup, M. H. (2014). Lineage sorting in apes. Annual Review of Genetics, 48, 519-535.

Martin, S. H., Davey, J. W., \& Jiggins, C. D. (2014). Evaluating the use of ABBA-BABA statistics to locate introgressed loci. Molecular Biology and Evolution, 32(1), 244-257.

Martin, S. H., Davey, J. W., Salazar, C., \& Jiggins, C. D. (2019). Recombination rate variation shapes barriers to introgression across butterfly genomes. PLoS Biology, 17(2), e2006288.

Martin, S. H., \& Van Belleghem, S. M. (2017). Exploring evolutionary relationships across the genome using topology weighting. Genetics, 206(1), 429-438.

Matthey-Doret, R., \& Whitlock, M. C. (2019). Background selection and FST: consequences for detecting local adaptation. Molecular Ecology. doi: 10.1111/mec.15197.

McVicker, G., Gordon, D., Davis, C., \& Green, P. (2009). Widespread genomic signatures of natural selection in hominid evolution. PLoS Genetics, 5(5), e1000471.

Meisel, R. P., \& Connallon, T. (2013). The faster-X effect: integrating theory and data. Trends in genetics, 29(9), 537-544.

Munch, K., Nam, K., Schierup, M. H., \& Mailund, T. (2016). Selective sweeps across twenty millions years of primate evolution. Molecular Biology and Evolution, 33(12), 3065-3074.

Nachman, M. W., \& Payseur, B. A. (2012). Recombination rate variation and speciation: theoretical predictions and empirical results from rabbits and mice. 
Philosophical Transactions of the Royal Society B: Biological Sciences, 367(1587), 409-421.

Nadachowska-Brzyska, K., Burri, R., Olason, P. I., Kawakami, T., Smeds, L., \& Ellegren, H. (2013). Demographic divergence history of pied flycatcher and collared flycatcher inferred from whole-genome re-sequencing data. PLoS Genetics, 9(11), e1003942.

Nadeau, N. J., Whibley, A., Jones, R. T., Davey, J. W., Dasmahapatra, K. K., Baxter, S. W., . . . Blaxter, M. L. (2012). Genomic islands of divergence in hybridizing Heliconius butterflies identified by large-scale targeted sequencing. Philosophical Transactions of the Royal Society B: Biological Sciences, 367(1587), 343-353.

Neale, D. B., \& Ingvarsson, P. K. (2008). Population, quantitative and comparative genomics of adaptation in forest trees. Current opinion in plant biology, 11(2), 149-155.

Neale, D. B., \& Kremer, A. (2011). Forest tree genomics: growing resources and applications. Nature Reviews Genetics, 12(2), 111.

Nielsen, R., Korneliussen, T., Albrechtsen, A., Li, Y., \& Wang, J. (2012). SNP calling, genotype calling, and sample allele frequency estimation from new-generation sequencing data. PloS One, 7(7), e37558.

Nordborg, M., Hu, T. T., Ishino, Y., Jhaveri, J., Toomajian, C., Zheng, H., . . Goyal, R. (2005). The pattern of polymorphism in Arabidopsis thaliana. PLoS Biology, 3(7), e196.

Nosil, P., Funk, D. J., \& Ortiz-Barrientos, D. (2009). Divergent selection and heterogeneous genomic divergence. Molecular Ecology, 18(3), 375-402.

Pamilo, P., \& Nei, M. (1988). Relationships between gene trees and species trees. Molecular Biology and Evolution, 5(5), 568-583.

Patterson, N., Price, A. L., \& Reich, D. (2006). Population structure and eigenanalysis. PLoS Genetics, 2(12), e190.

Pease, J. B., \& Hahn, M. W. (2013). More accurate phylogenies inferred from lowrecombination regions in the presence of incomplete lineage sorting. Evolution, 67(8), 2376-2384.

Phung, T. N., Huber, C. D., \& Lohmueller, K. E. (2016). Determining the effect of natural selection on linked neutral divergence across species. PLoS Genetics, 12(8), e1006199.

Prüfer, K., Munch, K., Hellmann, I., Akagi, K., Miller, J. R., Walenz, B., . . Winer, R. (2012). The bonobo genome compared with the chimpanzee and human genomes. Nature, 486(7404), 527.

Ravinet, M., Faria, R., Butlin, R., Galindo, J., Bierne, N., Rafajlović, M., . . W Westram, A. (2017). Interpreting the genomic landscape of speciation: a road map for finding barriers to gene flow. Journal of Evolutionary Biology, 30(8), 1450-1477.

Ravinet, M., Yoshida, K., Shigenobu, S., Toyoda, A., Fujiyama, A., \& Kitano, J. (2018). The genomic landscape at a late stage of stickleback speciation: High genomic divergence interspersed by small localized regions of introgression. PLoS Genetics, 14(5), e1007358.

Sankararaman, S., Mallick, S., Dannemann, M., Prüfer, K., Kelso, J., Pääbo, S., . . . Reich, D. (2014). The genomic landscape of Neanderthal ancestry in present-day humans. Nature, 507(7492), 354. 
1011 Sankararaman, S., Mallick, S., Patterson, N., \& Reich, D. (2016). The combined

1012

1013

1014

1015

1016

1017

1018

1019

1020

1021

1022

1023

1024

1025

1026

1027

1028

1029

1030

1031

1032

1033

1034

1035

1036

1037

1038

1039

1040

1041

1042

1043

1044

1045

1046

1047

1048

1049

1050

1051

1052

1053

1054

1055

1056

1057

1058 landscape of Denisovan and Neanderthal ancestry in present-day humans. Current Biology, 26(9), 1241-1247.

Scally, A., Dutheil, J. Y., Hillier, L. W., Jordan, G. E., Goodhead, I., Herrero, J., . . Marques-Bonet, T. (2012). Insights into hominid evolution from the gorilla genome sequence. Nature, 483(7388), 169.

Schiffels, S., \& Durbin, R. (2014). Inferring human population size and separation history from multiple genome sequences. Nature Genetics, 46(8), 919.

Schumer, M., Cui, R., Powell, D. L., Rosenthal, G. G., \& Andolfatto, P. (2016). Ancient hybridization and genomic stabilization in a swordtail fish. Molecular Ecology, 25(11), 2661-2679.

Seehausen, O., Butlin, R. K., Keller, I., Wagner, C. E., Boughman, J. W., Hohenlohe, P. A., . . . Brännström, ̊. (2014). Genomics and the origin of species. Nature Reviews Genetics, 15(3), 176.

Siewert, K. M., \& Voight, B. F. (2017). Detecting long-term balancing selection using allele frequency correlation. Molecular biology and evolution, 34(11), 2996-3005.

Smith, J. M., \& Haigh, J. (1974). The hitch-hiking effect of a favourable gene. Genetics Research, 23(1), 23-35.

Stamatakis, A. (2014). RAxML version 8: a tool for phylogenetic analysis and post-analysis of large phylogenies. Bioinformatics, 30(9), 1312-1313.

Stankowski, S., Chase, M. A., Fuiten, A. M., Rodrigues, M. F., Ralph, P. L., \& Streisfeld, M. A. (2019). Widespread selection and gene flow shape the genomic landscape during a radiation of monkeyflowers. PLoS Biology, 17(7), e3000391.

Tarailo-Graovac, M., \& Chen, N. (2009). Using RepeatMasker to identify repetitive elements in genomic sequences. Current Protocols in Bioinformatics, 25(1), 4.10. 11-14.10. 14.

Turner, T. L., Hahn, M. W., \& Nuzhdin, S. V. (2005). Genomic islands of speciation in Anopheles gambiae. PLoS Biology, 3(9), e285.

Tuskan, G. A., Difazio, S., Jansson, S., Bohlmann, J., Grigoriev, I., Hellsten, U., . . . Salamov, A. (2006). The genome of black cottonwood, Populus trichocarpa (Torr. \& Gray). Science, 313(5793), 1596-1604.

Van Doren, B. M., Campagna, L., Helm, B., Illera, J. C., Lovette, I. J., \& Liedvogel, M. (2017). Correlated patterns of genetic diversity and differentiation across an avian family. Molecular Ecology, 26(15), 3982-3997.

Vicoso, B., \& Charlesworth, B. (2006). Evolution on the X chromosome: unusual patterns and processes. Nature Reviews Genetics, 7(8), 645.

Vijay, N., Weissensteiner, M., Burri, R., Kawakami, T., Ellegren, H., \& Wolf, J. B. (2017). Genomewide patterns of variation in genetic diversity are shared among populations, species and higher-order taxa. Molecular Ecology, 26(16), 4284-4295.

Wang, B., Mojica, J. P., Perera, N., Lee, C.-R., Lovell, J. T., Sharma, A., . . Rokhsar, D. S. (2019). Ancient polymorphisms contribute to genome-wide variation by long-term balancing selection and divergent sorting in Boechera stricta. Genome Biology, 20(1), 126.

Wang, J., Street, N. R., Scofield, D. G., \& Ingvarsson, P. K. (2016a). Natural selection and recombination rate variation shape nucleotide polymorphism across the genomes of three related Populus species. Genetics, 202(3), 1185-1200. 
1059 Wang, J., Street, N. R., Scofield, D. G., \& Ingvarsson, P. K. (2016b). Variation in linked selection and recombination drive genomic divergence during allopatric speciation of European and American aspens. Molecular Biology and Evolution, 33(7), 1754-1767.

Weng, J. K. (2014). The evolutionary paths towards complexity: a metabolic perspective. New Phytologist, 201(4), 1141-1149.

Wolf, J. B., \& Ellegren, H. (2017). Making sense of genomic islands of differentiation in light of speciation. Nature Reviews Genetics, 18(2), 87.

$\mathrm{Wu}, \mathrm{C}$. I. (2001). The genic view of the process of speciation. Journal of Evolutionary Biology, 14(6), 851-865.

Yin, T., DiFazio, S. P., Gunter, L. E., Zhang, X., Sewell, M. M., Woolbright, S. A., . . Wang, M. (2008). Genome structure and emerging evidence of an incipient sex chromosome in Populus. Genome Research, 18(3), 422-430.

\section{Data Accessibility Statement}

Raw whole genome resequencing data generated for this study have been deposited in the NCBI short read archive under accession number PRJNA576115

\section{Author Contributions}

J.W. conceived the study, analyzed the data and wrote the manuscript. E.J.P. provided the materials of $P$. davidiana used in this study. N.R.S., J.L., P.K.I. read and commented on the manuscript. All authors approved the final manuscript. 
1083

1084

1085

1086

1087

1088

1089

1090

1091

1092

1093

1094

1095

1096

1097

1098

1099

1100

1101

1102

1103

1104

1105

1106

1107

1108

1109

1110

1111

1112

1113

1114

1115

1116

1117

1118

1119

1120

1121

1122

1123

1124

1125

1126

1127

1128

1129

1130

1131

1132

\section{Figure legends:}

Figure 1. Phylogenetic and population genetic analyses of four Populus species. (A)

Sampling locations (black circle) of eight individuals from each of the four Populus

species included in this study. Species ranges for $P$. tremula, $P$. davidiana, $P$.

tremuloides and $P$. trichocarpa are indicated by red, green, blue and purple shading, respectively. (B) Maximum-likelihood phylogenetic tree reconstructed based on complete chloroplast sequences. Color scheme for the four species is the same in A-C. (C) Historical effective population size of the four Populus species inferred using MSMC v2 based on sets of eight haplotypes, with solid lines representing medians and shading representing \pm standard deviation calculated across pairs of haplotypes. Yellow bar indicates Early Pleistocene cooling; Glacial and interglacial periods of the Late and Middle Pleistocene are indicated by dark and light grey bars, respectively; black bar indicates the period of Last Glacial Maximum (LGM). (D) Schematic of demographic scenarios of the three aspen species modeled using fastsimcoal2. The ancestral population is shown in light and dark grey respectively for different ancestral lineages. $P$. tremula is in red, $P$. davidiana is in green, and $P$. tremuloides is in blue. The arrows indicate the per generation migration rate $(m)$ between species. Estimated divergence time, effective population size, and gene flow are detailed in Supplementary Table S7.

\section{Figure 2. Genome-wide landscape of genetic diversity and divergence within and} between species. (A) Chromosomal landscape of (a) the density of coding sequences (CD); (b) nucleotide diversity $(\pi)$; (c) recombination rate $(\rho)$; (d) the relative measure of genetic divergence $\left(F_{\mathrm{ST}}\right)$ and $(\mathrm{e})$ the absolute measure of genetic divergence $\left(\mathrm{d}_{\mathrm{xy}}\right)$. $(\mathrm{B})$ Distribution of correlation coefficients (Spearman's $\rho$ ) shown as violin plots for population summary statistics characterizing genomic features (neutral mutation rate $\mu$ ) and variation within $(\pi, \rho)$ and between species $\left(F_{\mathrm{ST}}, \mathrm{d}_{\mathrm{xy}}\right)$ calculated at $100 \mathrm{Kbp}$ windows. Subscripts 'i, j' symbolize all possible combinations of correlations between two species $i=1 \ldots(n-1)$ and $j=(i+1) \ldots n$ for within-species measures; Capital letters ' $I, J$ ' symbolize inter-species statistics. Correlations exclude pseudo-replicated species comparisons. Detailed information can be found in Supplementary Table S11.

Figure 3. Heterogeneous distribution of phylogenies in three aspen species. Chromoplots for 19 chromosomes show the distribution of three possible rooted phylogenetic relationships inferred from $100 \mathrm{Kbp}$ genomic regions for $P$. tremula $(P$. tra), P. davidiana ( $P$. dav) and $P$. tremuloides ( $P$. trs), with $P$. trichocarpa as outgroup species. The colored vertical bars represent the windows with complete monophyly of the three alternative topologies as shown in the lower right, where the proportion of the three topologies in $100 \mathrm{Kbp}$ and $10 \mathrm{Kbp}$ (in parenthesis) across the genome are also shown. Across all chromosomes, the $D$ statistic generally tends toward positive values, indicating ancient introgression between $P$. tra and $P$. trs may have been occurring across the genome.

Figure 4. Widespread impact of linked selection. Relationship between recombination rate (blue), coding density (red) and (A) genetic diversity, (B) $F_{\mathrm{ST}}$, (C) $\mathrm{d}_{\mathrm{xy}},(\mathrm{D})$ incomplete lineage sorting (ILS), (E) weighting of the 'species' tree ([P. tra, $P$. $d a v], P$. trs $)$ ) and (F) the estimated admixture proportion $\left(f_{\mathrm{d}}\right)$ between $P$. tra and P. trs. Quantile binning is for visualization. The points and error bars indicate the means and $1.96 \times$ standard errors. Statistical tests were performed on the unbinned data and detailed correlation coefficients are shown in supplementary Table S15-Table S22. 
1133

1134 Figure 5. Identification of positive selection. (A) Positive selection analysis by

1135

1136

1137

1138

1139

1140

1141

1142

1143

1144

1145

1146

1147

1148

1149

1150

1151

1152

1153

1154

1155

1156

1157

1158

1159

1160

1161

1162

SweepFinder2 reveals windows that are candidates for being under positive selection in the three aspen species: P. tremula (P. tra), P. davidiana $(P$. dav) and $P$. tremuloides $(P$. trs). Horizonal red line indicates the cut-off of composite likelihood ratio (CLR) statistics. (B) The Venn diagram represents shared and unique selected windows detected in the three species. (C) Comparison of genetic diversity, recombination rate, $F_{\mathrm{ST}}, \mathrm{d}_{\mathrm{xy}}$, and average weightings of the 'species' topology between candidate regions under positive selection (red boxes) and genomic background (grey boxes). (D) Comparison of incomplete lineage sorting (ILS) and the estimated admixture proportion $\left.\left(f_{\mathrm{d}}\right)\right)$ between candidate regions under positive selection (red boxes) and genomic background (grey boxes). Asterisks designate significant differences between candidate positive selected regions and the rest of genomic regions by Mann-Whitney $U$-test $\left({ }^{\text {n.s. }}\right.$ Not significant; ${ }^{*} P$ value $<0.01 ;{ }^{* *} P$ value $<0.001 ;{ }^{* * *} P$ value $\left.<1 \mathrm{e}-4\right)$.

Figure 6. Identification of long-term balancing selection. (A) Signals of balancing selection across all chromosomes in the three aspen species: $P$. tremula (P. tra), $P$. davidiana $(P$. dav) and $P$. tremuloides $(P . t r s)$. Horizonal red line indicates the cut-off of the $\beta$ statistics. Only the signals detected in all three aspen species (red dots) were considered as being under long-term balancing selection. (B) The Venn diagram represents shared and unique selected SNPs detected in the three species. (C) Comparison of genetic diversity, recombination rate, $F_{\mathrm{ST}}, \mathrm{d}_{\mathrm{xy}}$, and average weightings of the 'species' topology between candidate regions under long-term balancing selection (red boxes) and genomic background (grey boxes). (D) Comparison of incomplete lineage sorting (ILS) and the estimated admixture proportion $\left(f_{\mathrm{d}}\right)$ ) between candidate regions under long-term balancing selection (red boxes) and genomic background (grey boxes). Asterisks designate significant differences between candidate balancing selected regions and the rest of genomic regions by Mann-Whitney $U$-test $\left({ }^{\text {n.s. }}\right.$ Not significant; ${ }^{*} P$ value $<0.01 ;{ }^{* *} P$ value $<0.001 ;{ }^{* * *} P$ value $\left.<1 \mathrm{e}-4\right)$. 
A

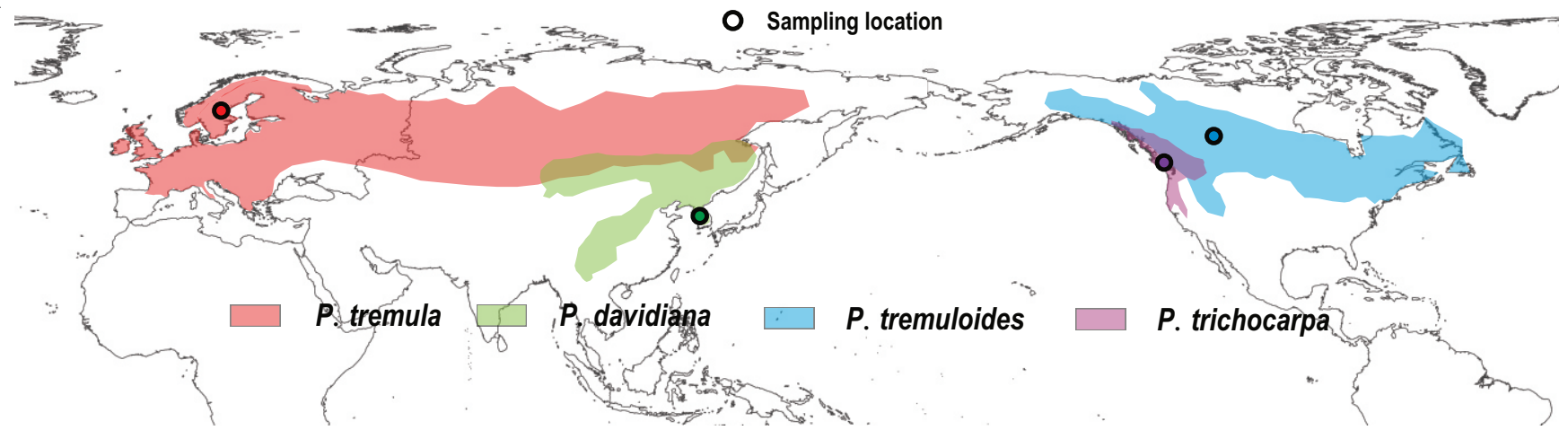

B

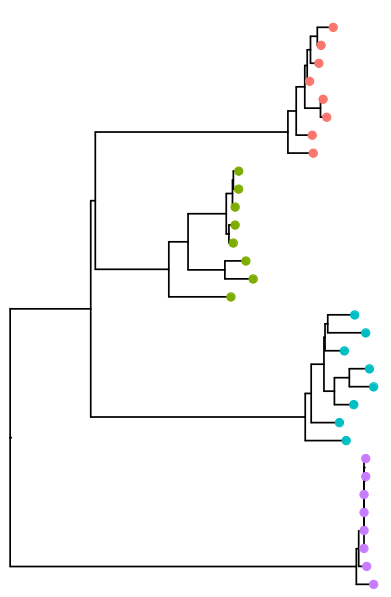

0.03
C

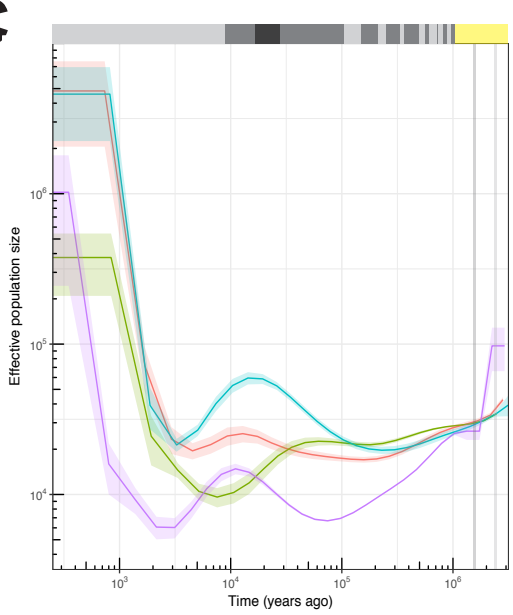

D

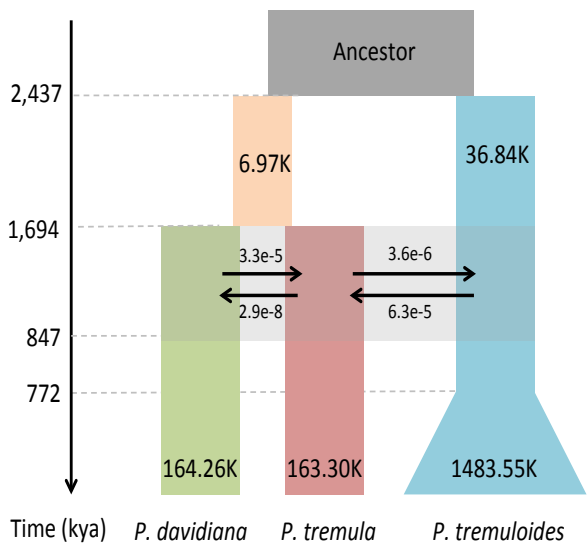


A

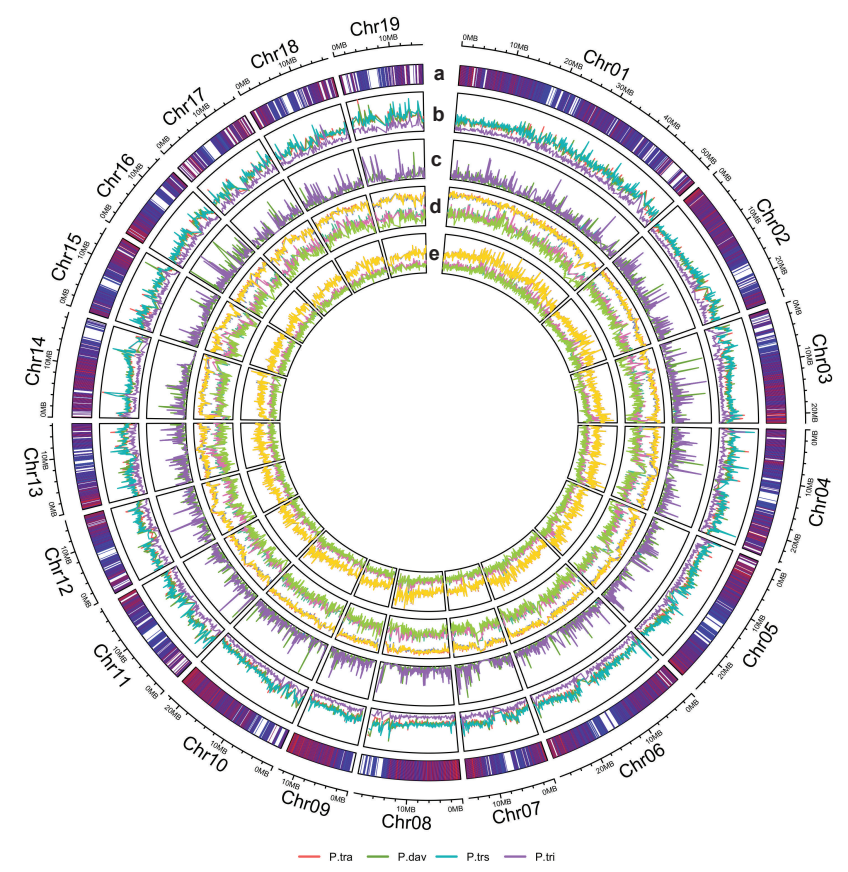

B

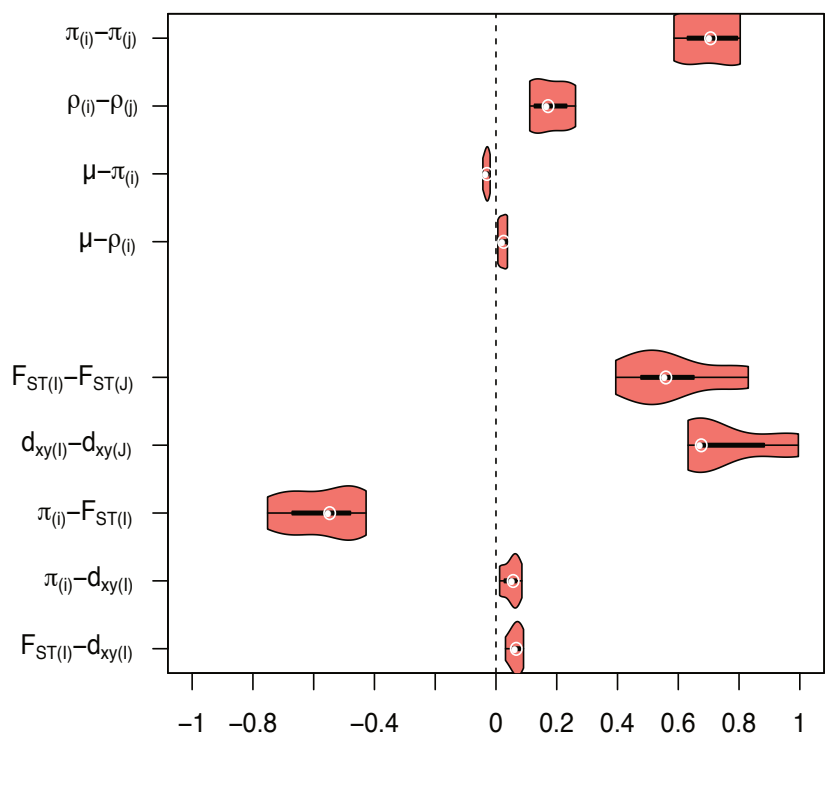




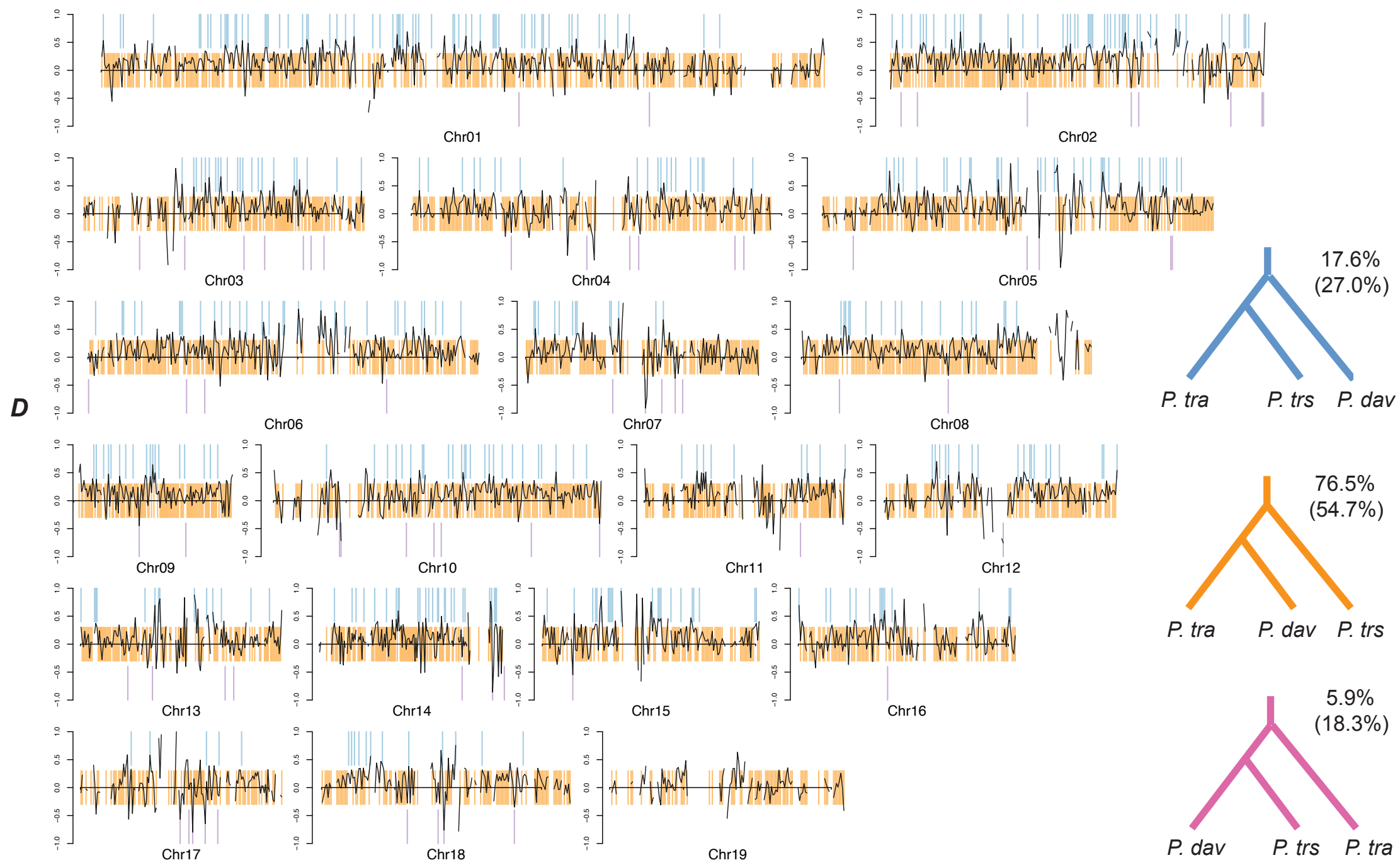




\section{Coding density}

A

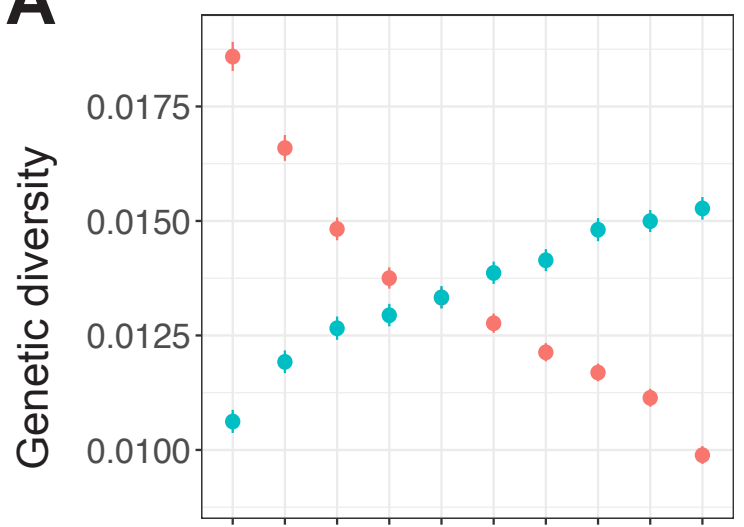

D

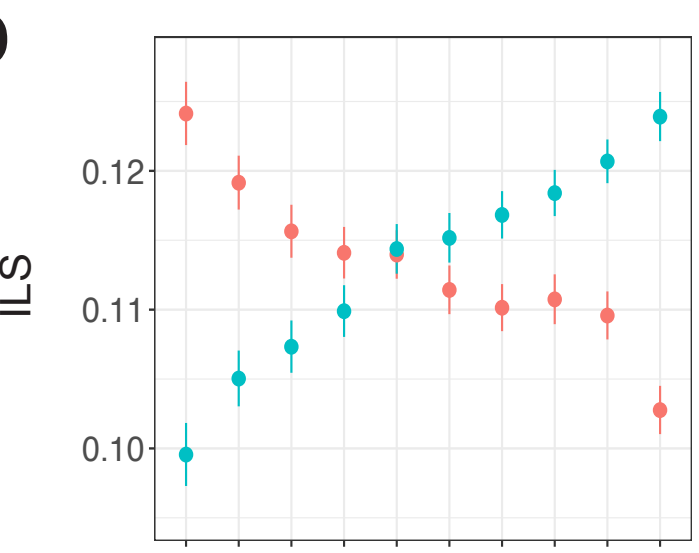

olo olo olo olo olo olo olo olo olo olo

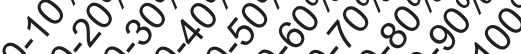
,
B

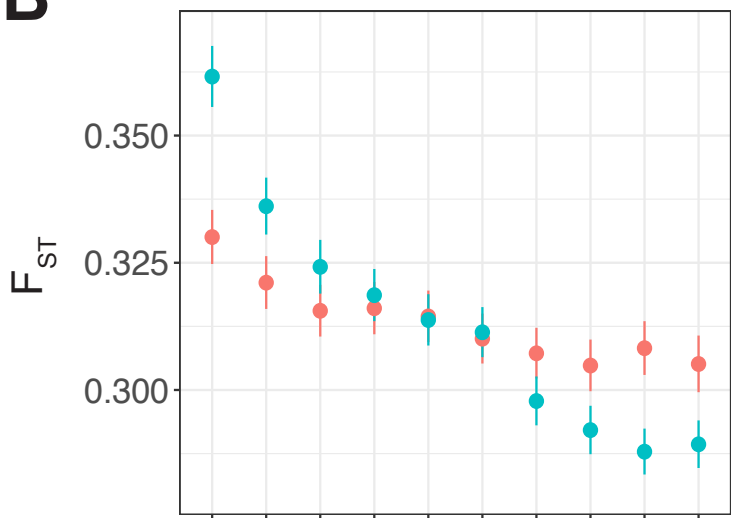

E

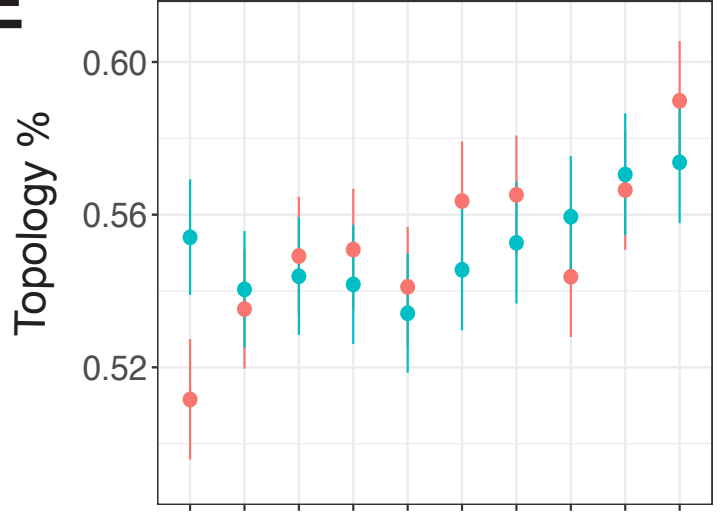

olo olo olo olo olo olo olo olo olo olo $0,5,5050,0,0,0,0$

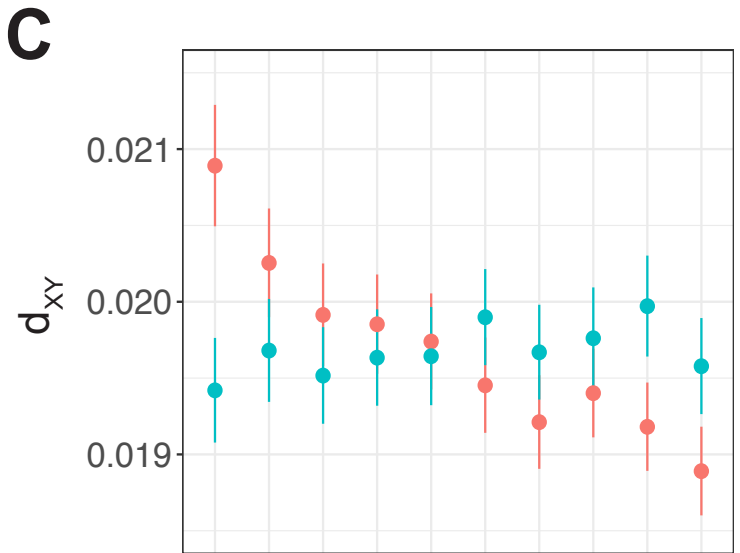

$\mathbf{F}$

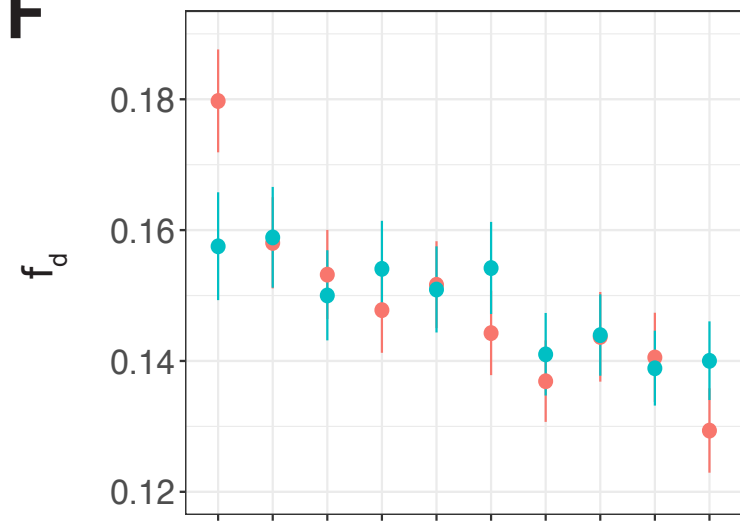

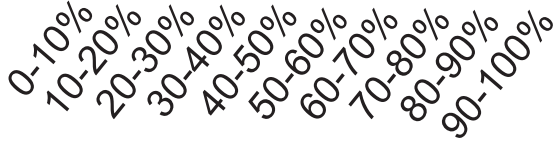

Percentile of coding density and recombination rate 


\section{A}
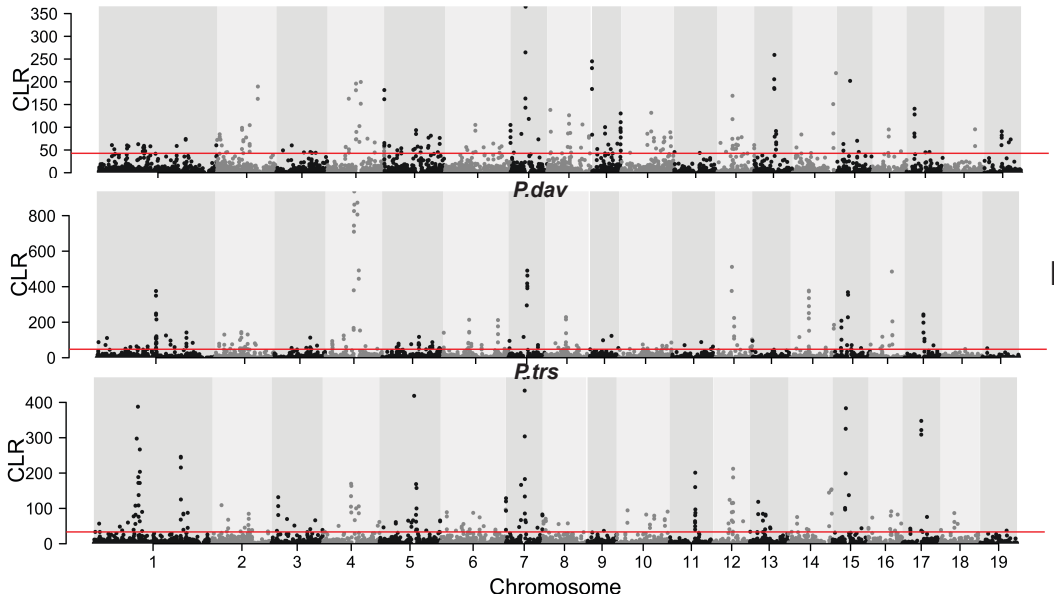

Chromosome

C

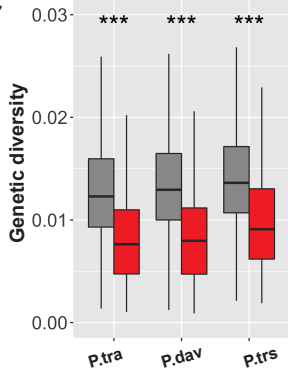

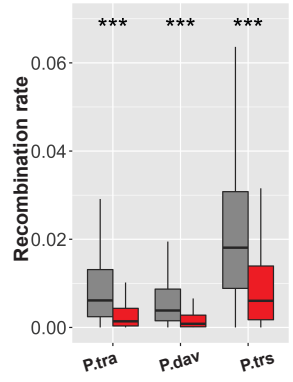

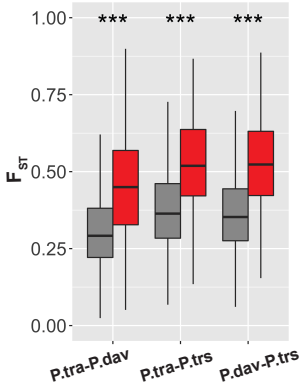

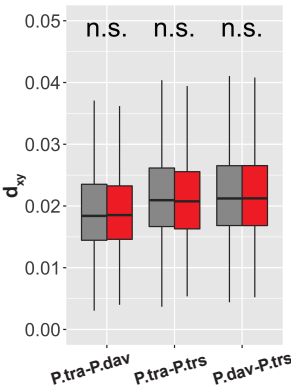

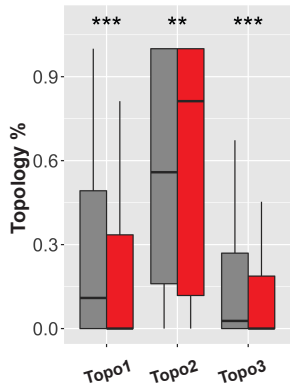

TOPO1 TOPO2 TOPO3

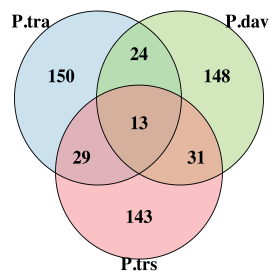

D

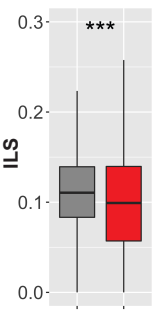

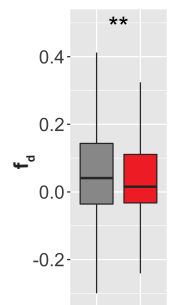




\section{A}

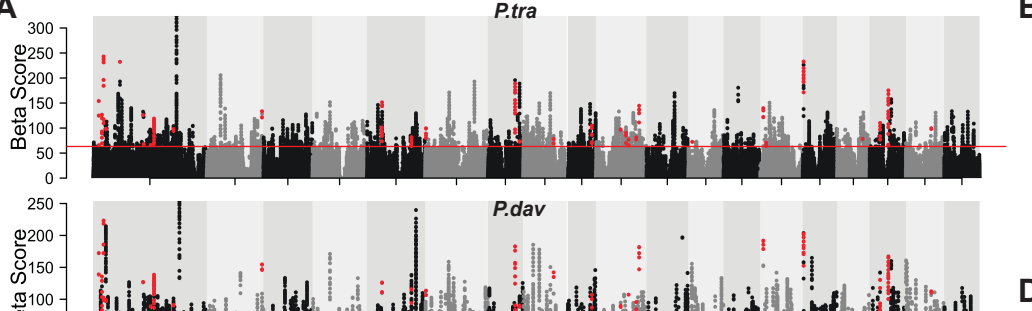

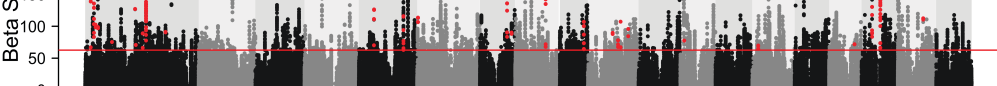
(150-

c

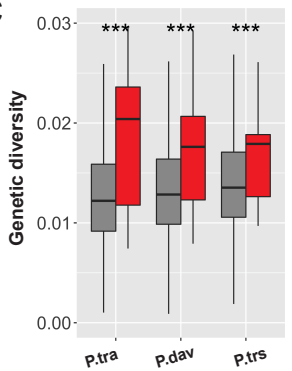

B
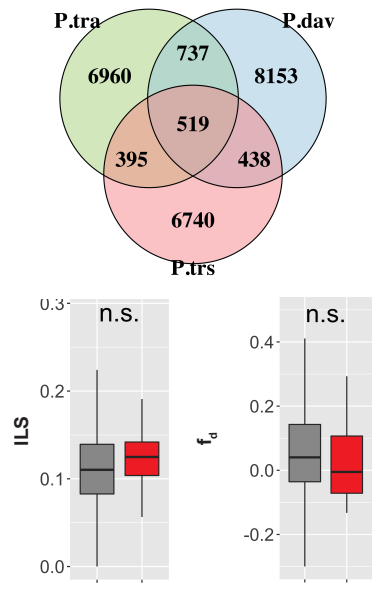

n.s. n.s. n.s.

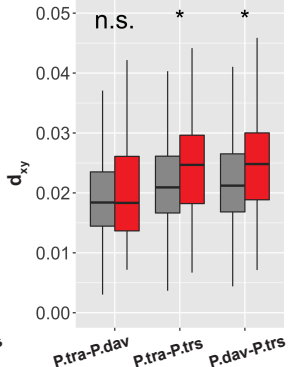

